DOI: $10.3901 / J M E .2021 .06 .026$

\title{
结构疲劳百年研究的回顾与展望
}

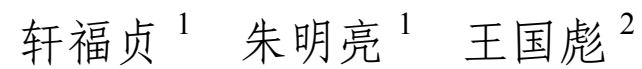 \\ (1. 华东理工大学机械与动力工程学院 上海 200237; \\ 2. 国家自然科学基金委员会工程与材料科学部 北京 100085)
}

\begin{abstract}
摘要: 疲劳是机械结构最普遍的失效模式之一。自 1854 年第一次提出 “Fatigue” (疲劳)概念以来, 相关研究已有 160 余年的 历程, 逐步形成了以疲劳研究为基础的机械结构强度理论与技术, 推动机械结构从经验设计走向安全设计。本文回顾了结构 疲劳研究的缘起及发展历程, 总结了典型失效案例对疲劳基础研究的促进作用和里程碑式成果, 基于文献统计分析了最近 50 年本领域的代表性进展、研究热点与发展趋势。研究表明, 尽管经历了百余年的不解努力, 疲劳极限、损伤易感基因、裂尖 主控机制、蠕变-疲劳交互和安全系数的物理本质等仍是困扰人们的难题。数据科学和大数据技术的兴起, 为突破数据驱动的 疲劳寿命预测方法、诠释疲劳损伤物理机制和建立极端条件下的高端装备疲劳可靠性设计技术提供了新的途径。
\end{abstract}

关键词: 疲劳; 断裂; 蠕变; 损伤机制; 寿命预测; 结构设计

中图分类号: TG115

\section{Retrospect and Prospect on Century-long Research of Structural Fatigue}

\author{
XUAN Fuzhen $^{1} \quad$ ZHU Mingliang $^{1} \quad$ WANG Guobiao ${ }^{2}$ \\ (1. School of Mechanical \& Power Engineering, \\ East China University of Science \& Technology, Shanghai 200237; \\ 2. Department of Engineering and Materials Sciences, \\ National Natural Science Foundation of China, Beijing 100085)
}

\begin{abstract}
As one of the most common failure modes of mechanical structures, fatigue has been researched for more than 160 years since the term "fatigue" was first proposed in 1854, and the fatigue knowledge has laid a solid foundation for theory and modern technology in mechanical strength areas, and has promoted structural design from experience to safety concept. The paper presented a retrospect on the origin and development, and a summary of the milestones achieved in fatigue research that was driven by typical engineering failure cases. A thorough literature data mining was carried out to illustrate the highlighted achievements, hot topics, and new research trends in the past 50 years. The work showed that the fatigue limit, microstructural genetics, crack-tip driving force, creep-fatigue interaction, and safety factor, were still difficult topics to be solved, though explored for century-long period. The work also indicates that, the emergence of data science and big data technology can provide new pathways to make breakthroughs and establishments in data-driven fatigue life prediction, physical mechanisms of fatigue damage interpretation, and fatigue reliability design of high-end equipment at extreme working conditions.
\end{abstract}

Key words: fatigue; fracture; creep; damage mechanisms; life prediction; structural design

1 疲劳研究的起源与演化

\section{1 蒸汽动力催生结构失效}

1763 年瓦特改良蒸汽机, 成为机器动力革命的
重要推动力, 开启了人类大规模运用火车、轮船等 动力机械时代。相比于早期的水力、人力驱动, 蒸 汽驱动的机器运转速度、频率和载荷水平都大幅提 升, 以高速、重载为特征的大工业触发大量机器失 效。世界各国相继成立专门机构或国际组织颁布统 一标准以应对机器失效问题。例如, 1817 年, 英国 成立专门委员会以防止蒸汽船爆炸的危险和破坏;

20210121 收到初稿, 20210203 收到修改稿 
1833 年, 英国成立曼彻斯特蒸汽锅炉保险公司, 检 查和确保锅炉免受爆炸造成的损害; 1911 年, ASME 成立 “蒸汽锅炉和压力容器建造和在役维护标准规 范” 委员会; 1946 年, 美国测试与材料协会成立疲 劳专业委员会; 1979 年, 我国在国家劳动总局锅炉 局下建立锅炉压力容器检测中心站。1911 年左右, ASME 发布了世界上第一部标准; 1939 年, 英国编 写熔焊压力容器的标准和规范; 1982 年, 我国正式 颁布第一部压力容器部级标准。

动力机械具有的载荷不恒定、随机波动和随时间 变化等因素构成了疲劳失效的典型特征。纵观疲劳研 究的历史, 疲劳问题的提出经历了漫长的探索过程。 1837 年, ALBERT ${ }^{[1]}$ 设计传动链的测试装置, 发表第 一篇有关疲劳的论文; 1839 年, PONCELET ${ }^{[2]}$ 在巴黎 大学报告中描述金属会 “累”; 1842 年, RANKINE ${ }^{[3]}$ 在研究轮轨失效中意识到应力集中的重要性; 1849 年, HODGKINSON ${ }^{[4]}$ 提出了 “结构上连续变化的载 荷的影响, 以及此类结构能耐载到什么程度而不影响 安全” 的问题; 1854 年, BRAITHWAITE ${ }^{[5]}$ 正式提出 了 “Fatigue” (疲劳)的概念。疲劳的初期研究体现着 人们对结构失效现象的浅层认识。

相对而言, 有关疲劳的科学知识在大学和课堂 传授滞后于工业界的研究。例如, 1794 年, 拿破仑 支持成立世界上第一个工程教育机构一巴黎技术 学院, 开设机构学和应用数学等课程; 1846 年, 在 德国教育家雷腾巴赫倡导下, 卡尔斯鲁厄技术学校 设置机械系, 进一步形成机械设计课程体系; 1847 年, 英国成立世界上第一个工程学会, 即英国机械 工程学会; 1861 年, 德国 REULEAUX 出版《机械 设计者》, 标志着机械设计学脱离应用力学; 1924 年, 英国国家物理实验室的 $\mathrm{GOUGH}^{[6]}$ 出版第一部 疲劳专著《金属的疲劳》; 1980 年, 高镇同教授 ${ }^{[7]}$ 出版我国第一本疲劳方面的专著《疲劳性能测试》。

\section{2 结构失效驱动疲劳研究}

人们对疲劳的研究是从对失效事故的调查开始 的。1 842 年, 法国凡尔赛火车轮轴断裂, 火车出轨 起火, 约 60 人死亡, 此后发生了系列火车零部件破 坏事故, 促使人们开始重视并开展相关研究。那一 时期的人们重点关注如何再现破坏过程, 为此开展 了结构疲劳试验。例如, ALBERT ${ }^{[1]}$ 开展了采矿提 升机链条加载试验, FAIRBAIRN ${ }^{[8]}$ 开展了梁的弯曲 疲劳试验, 并从试验结果中认识到结构存在安全载 荷。然而, 结构疲劳试验对破坏规律认识不足, 从 试样角度认识疲劳成为人们的研究重点, 这一研究 思路最终促进了疲劳极限和 $S-N$ 曲线的提出。
1954 年, 首架英国彗星号(Comet)喷气客机坠 落地中海, 68 人死亡; 事故源于飞机窗户角的高应 力集中区导致的疲劳。1957-1958 年间, 连续发生 了多起因结构疲劳失效引起的美军 B-47 轰炸机空 中解体失事事件。20 世纪 50 年代发生的多起疲劳 事故, 促使人们更加关注缺口疲劳失效问题, 推动 了应变-疲劳理论与方法的发展与进步, 应变-疲劳 成为除应力-疲劳外的重要研究领域之一。

对疲劳裂纹扩展规律的认识也起源于结构失效 事故 ${ }^{[9]}$ 。二战期间, 美国 2500 余艘全焊接自由轮 (Liberty ship)中, 700 余艘发生焊接接头原始缺陷引起 的断裂事故 ${ }^{[10]}$ 。1967-1969 年间, 美国空军 F-111 可 变后掠翼战机多达 4 架次由于机翼枢纽加工原始裂纹 缺陷导致坠机事故 ${ }^{[11]}$ 。1977 年, 波音 707 货机在陆萨 卡发生水平机翼初始裂纹扩展诱发的尾翼断裂事故。 这些失效事故使人们对缺陷和裂纹有了深刻的认识, 缺陷处裂纹萌生及扩展行为与结构断裂密切相关, 而 有关裂纹扩展规律的理论研究受益于 20 世纪 60 年代 以来断裂力学的发展。其中, IRWIN ${ }^{[12]}$ 于 1958 年基 于断裂力学原理, 研究了裂尖力学场的表征问题;

PARIS 与 ERDOGEN ${ }^{[13]}$ 于 1960 年发现疲劳裂纹扩展 的幂指数规律, 对疲劳裂纹扩展行为进行了理论分 析; ELBER ${ }^{[14]}$ 于 1970 年提出裂纹闭合的概念, 揭示 了应力比等因素对疲劳裂纹扩展行为的影响。

微观分析技术的进步促使人们开展疲劳失效损 伤机制的研究, 并分析其与静态拉伸破坏的差异。 静态破坏时材料具有明显的塑性变形, 失效时的载 荷超过了材料的断裂强度; 在循环载荷条件下, 虽 然材料承受的载荷低于屈服强度, 但经过多次往复 加载后, 会发生突然断裂, 这种突发性和低应力特 性, 成为结构抗疲劳设计的难题。此外, 不同疲劳 破坏模式的出现, 也推动着疲劳裂纹萌生与扩展机 制的研究不断向深度和广度拓展。

\section{3 疲劳研究支撑机械强度学}

针对结构的失效问题, 一方面, 人们关注如何 减小结构动应力, 降低振动与噪声, 提高设备的可 靠性, 催生了振动力学, 它以系统的平衡、转子动 力学、速度波动调节, 以及振动、隔振与噪声等问 题为主要内容, 相关研究促进了机械动力学的产生 和发展; 另一方面，人们考虑如何使材料更加 “健 壮”, 从而能使设备在有裂纹的情况下也能安全运 行, 产生了材料力学, 它以材料的破坏机理、结构 设计的安全准则、应力的准确表达, 以及考虑构件 几何形状的影响等为主要内容, 进一步发展成为损 伤力学、断裂力学, 进而产生了机械强度学, 支撑 
了强度理论的发展 ${ }^{[15]}$ 。机械动力学与机械强度学两 个学科的共同目标是统一的, 即解决机器的失效、 振动、可靠性和寿命预测等问题 ${ }^{[16]}$ 。

近百年来, 疲劳强度的基础研究支持了疲劳设 计技术的进步。图 1 表示了百年来结构设计技术随 疲劳基础研究的演化历程。疲劳设计技术经历了经 验类比设计、安全寿命设计、失效-安全设计、损伤 容限设计和超长寿命设计等五个阶段。18 世纪后, 人们以材料力学为基础进行经验类比设计; 20 世纪 50 年代后, 随着高周疲劳理论的发展与成熟, 线弹 性强度理论和安全系数引入后, 安全寿命是主要的 设计技术, 结构设计以无限寿命为目标; 20 世纪 60 年代, 随着低周瘦劳和局部应力应变理论的发展, 形成了失效-安全设计技术, 目的是允许构件失效而 不引起整个部件的失效; 20 世纪 70 年代后, 随着 疲劳断裂理论和无损检测技术的进步, 损伤容限设 计技术得到蓬勃发展, 美国空军于 1974 年制定损伤 容限设计规范(A-83444), 其最大的特点是允许结构 裂纹的存在, 通过描述裂纹扩展并与疲劳寿命进行 关联, 成为结构剩余寿命评价和预测的重要工具; 进入 21 世纪, 随着人们对超高周疲劳研究的深入, 超高周疲劳的断裂行为与材料的冶金和制造联系起 来, 考虑缺陷致裂进而发展超长寿命设计方法成为 研究热点, 以满足工程结构超长寿命的服役需求。

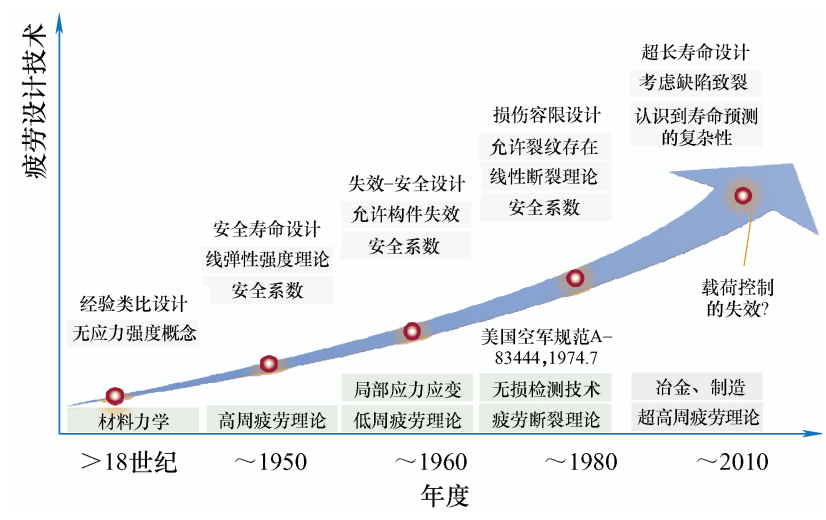

图 1 百年来疲劳设计技术随疲劳基础研究的演化历程

尽管疲劳设计技术得到了长足的发展与进步, 诸 如大型宽体客机和第四代增殖反应堆等新型高端装备 的发展对疲劳强度、损伤模式与寿命设计的需求仍然 迫切, 疲劳设计仍然是高端装备研发的重要使能技术。

\section{2 文献综合分析}

在百余年的疲劳研究中, 人们发表了大量的学 术论文和著作。通过对发表的文献进行综合分析, 总结具有重要影响的工作和关键研究领域, 解析和
认识疲劳研究的发展与演化, 从而透过热点洞悉该 领域的未来发展趋势。

\section{1 论文发表情况}

以 “Fatigue” 作为关键词, 在 Web of Science 数 据库中，共检索到学术论文 66521 篇(截至 2020 年 11 月)。图 2 所示为 1970-2020 年间疲劳领域研究论 文发表情况。可以看出, 论文发表数量逐年增加, 尤 其是近几年,每年的论文发表数量已经超过 3000 篇。 从增长趋势中分析发现, 论文数量基本呈现指数形式 稳步增长, 表明疲劳领域研究的热门度逐渐升高。对 检索到的论文按学科领域分类(图 3), 可见: 机械工 程、冶金工程和力学三个学科发表的论文占据论文总 数的 $64 \%$, 约 $44 \%$ 的论文可归属到材料科学综合学科 (由于同一篇论文可能属于多个学科门类, 故上述论 文总占比数量高于 $100 \%$ 。从论文发表的学术期刊分 布情况看 (图 4), International Journal of Fatigue (IJFatigue)、 Fatigue \& Fracture of Engineering Materials \& Structures (FFEMS) 和 Materials Science and Engineering A (MSEA)等期刊发表的论文数量分别为 4959 篇、2 093 篇和 1914 篇, 是疲劳领域的三大主 流期刊。从论文所属国家分布看(图 5), 美国发表的 论文数量最多, 达到 13998 篇, 中国(大陆)次之, 发 表 11065 篇, 而日本发表论文数量居于第三位, 达到 6859 篇。表明美国、中国和日本是国际上疲劳领域 研究最活跃的三个国家。

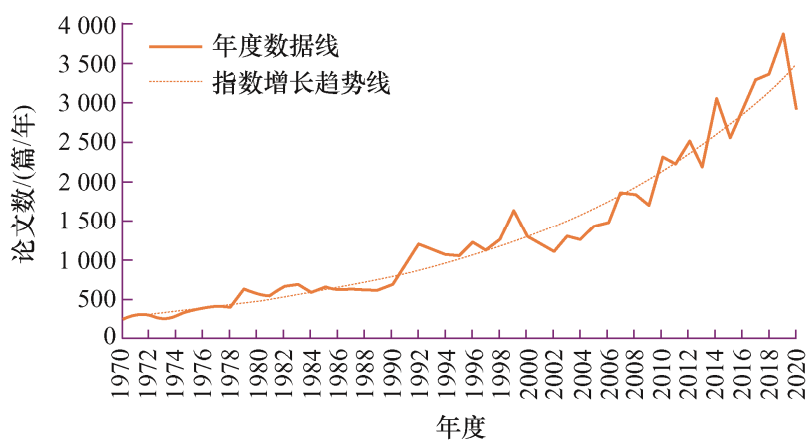

图 2 1970-2020 年间疲劳研究论文发表情况
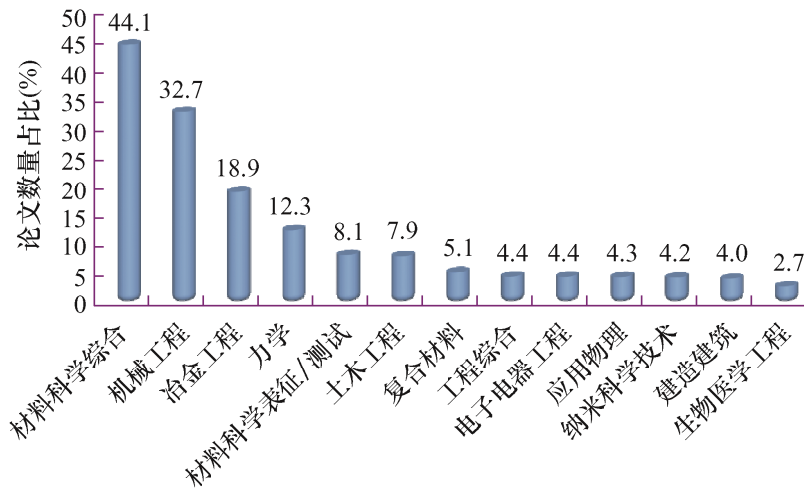

学科领域

图 3 1970-2020 年间疲劳研究论文按学科领域分布情况 


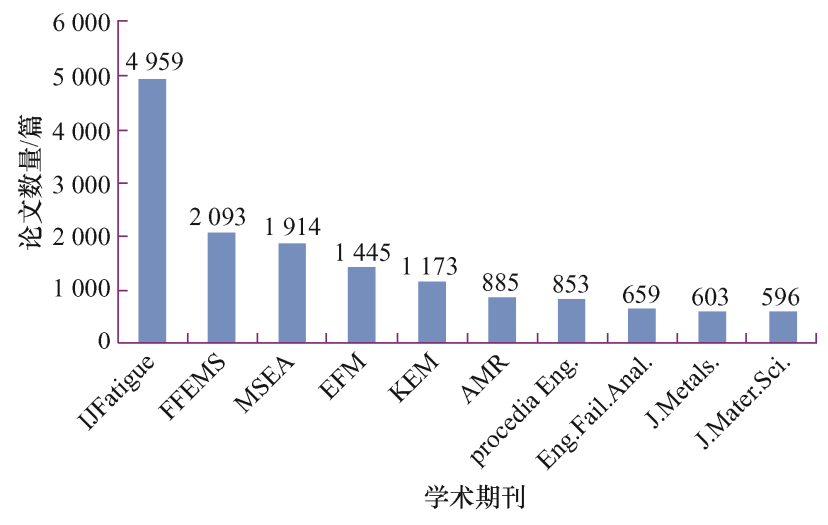

图 4 1970-2020 年间疲劳论文发表主要学术期刊分布

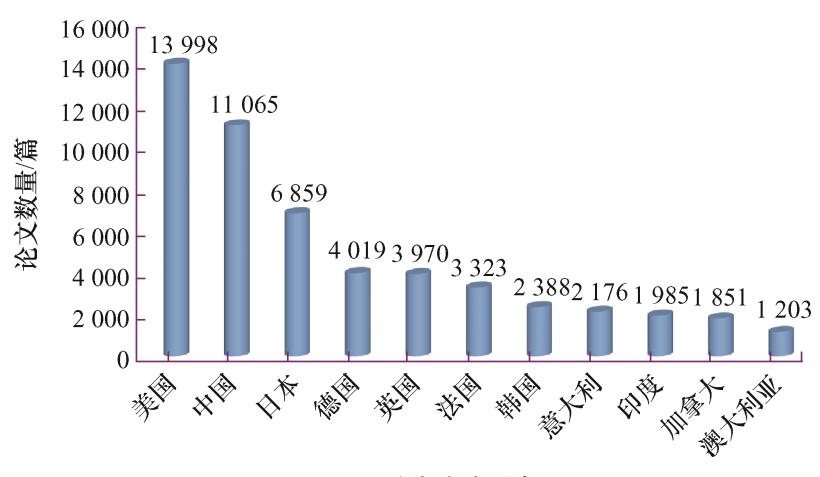

论文发表国家

图 51970 - 2020 年间疲劳研究论文发表国家分布情况

\section{2 重要影响工作分析}

为进一步分析近 50 年疲劳领域的重要影响工 作，从 1970-2020 年发表的 66521 篇论文中，选 取引用量靠前的 1000 篇论文进行分析, 这些论文 被认为是疲劳领域的高被引论文。图 6、图 7 和图 8 所示分别为 1000 篇论文的期刊分布、国家分布和 高被引作者。可以看出, IJFatigue、MSEA、FFEMS 和 Engineering Fracture Mechanics(EFM)是发表疲劳 高被引论文的主流期刊, 且 IJFatigue 的高被引论文 数量具有绝对优势，达到 182 篇; 美国、英国、日 本、德国和法国等 5 个发达国家是疲劳领域高被引 论文的主要产出国家, 中国紧随其后, 其中美国共 有 430 篇高被引论文, 占绝对优势, 表明对疲劳领 域的影响力大; RITCHIE R O、SURESH S、 MURAKAMI Y、LAZZARIN P、MUGHRABI H、 MCDOWELL D L、LIAW P K 和 SONSINO C M 等 是疲劳领域发表高被引论文数排名前八位的作者, 其中 RITCHIE R O 教授发表了 42 篇高被引论文, 可被认为是疲劳领域最具影响力的学者。此外, BATHIAS C、FATEMI A、LINDLEY T C、MILLER $\mathrm{K} J$ 和 TANAKA $K$ 等也是疲劳领域著名的学者。表 1 列出了被引次数排名前 20 的论文信息, 作为 Web of Science 数据库的补充, 表 1 中含有部分 Google
Scholar 的检索结果。可以看出, 被引次数最高的论 文为 ELBER 于 1970 年发表的有关裂纹闭合的论 文, 引用次数排名第 2 至 5 的论文, 均是 20 世纪 70 年代发表的论文, 其研究主题涉及复合材料疲劳 失效准则、金属疲劳应力-应变关系、疲劳裂纹扩展 的应力比效应和多轴疲劳理论。结合其他高被引论 文的研究主题, 可以看出, 在疲劳的基本理论和规 律、热点新材料的疲劳机制等方面的研究成果容易 引起高度关注。值得注意的是, 2010 年发表的石墨 烯纳米聚合物疲劳与断裂, 2012 年发表的增材制造 材料疲劳行为研究, 被引次数增加较快, 成为热点 新方向。

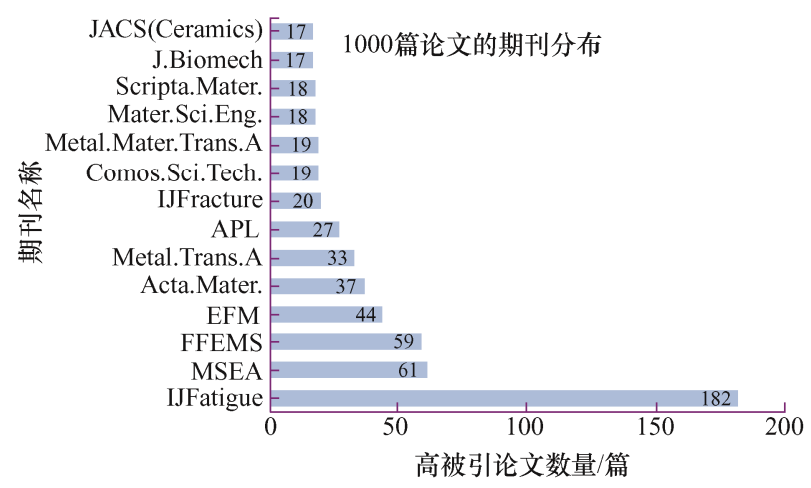

图 61970 -2020 年间疲劳领域高被引论文期刊分布

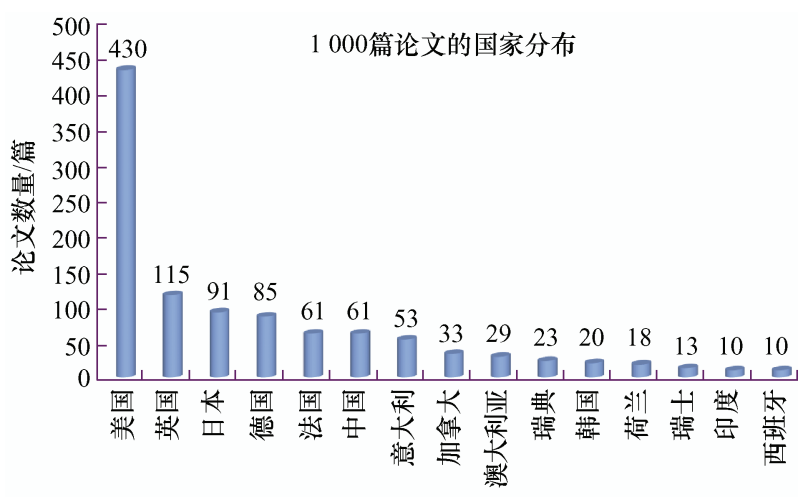

论文发表国家

图 $71970-2020$ 年间疲劳领域高被引论文国家分布

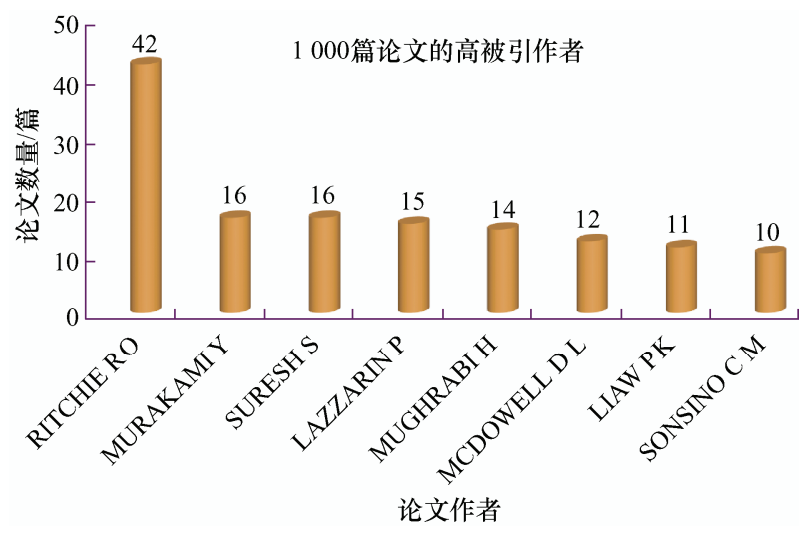

图 8 1970-2020 年间疲劳领域发表高被引论文作者 
表 $12970-2020$ 年间疲劳领域被引次数排名前 20 的论文列表

\begin{tabular}{|c|c|c|c|c|c|}
\hline 序号 & 论文题目 & 作者 & 期刊 & 刊文年份 & 引用数 \\
\hline 1 & Fatigue crack closure under cyclic tension & ELBER W & EFM & 1970 & 2507 \\
\hline 2 & $\begin{array}{l}\text { A Fatigue Failure Criterion for Fiber Reinforced } \\
\text { Materials }\end{array}$ & HASHIN Z, ROTEM A & J. Composite Materials & 1973 & 1482 \\
\hline 3 & Stress-strain function for fatigue of metals & $\begin{array}{l}\text { SMITH K N, WATSON P, } \\
\text { TOPPER T H }\end{array}$ & J. of Materials & 1970 & 1356 \\
\hline 4 & $\begin{array}{l}\text { The effect of stress ratio during crack propagation and } \\
\text { fatigue for } 2024-\mathrm{T} 3 \text { and } 7075-\mathrm{T} 6 \text { aluminum }\end{array}$ & WALKER K & ASTM STP462 & 1970 & 1107 \\
\hline 5 & $\begin{array}{c}\text { A Theory for Fatigue Failure under Multiaxial } \\
\text { Stress-Strain Conditions }\end{array}$ & BROWN M W, MILLER K J & $\begin{array}{l}\text { Proc. of Institution of } \\
\text { Mechanical Engineers }\end{array}$ & 1973 & 1050 \\
\hline 6 & $\begin{array}{c}\text { A critical plane approach to multiaxial fatigue damage } \\
\text { including out-of-phase loading }\end{array}$ & FATEMI A, SOCIE D F & FFEMS & 1988 & 953 \\
\hline 7 & $\begin{array}{l}\text { Cumulative fatigue damage and life prediction } \\
\text { theories: a survey of the state of the art for } \\
\text { homogeneous materials }\end{array}$ & FATEMI A, YANG L & IJFatigue & 1998 & 716 \\
\hline 8 & Stress corrosion and static fatigue of glass & $\begin{array}{l}\text { WIEDERHORN S M, BOLZ L } \\
\text { H }\end{array}$ & JACS(ceramics) & 1970 & 663 \\
\hline 9 & Fracture and fatigue in graphene nanocomposites & $\begin{array}{l}\text { RAFIEE M A, RAFIEE J, } \\
\text { SRIVASTAVA I, et al }\end{array}$ & Small & 2010 & 596 \\
\hline 10 & $\begin{array}{l}\text { Mechanisms of fatigue crack-propagation in metals, } \\
\text { ceramics and composites - role of crack tip shielding }\end{array}$ & RITCHIE R O & MSEA & 1988 & 539 \\
\hline 11 & $\begin{array}{l}\text { Effects of defects, inclusions and inhomogeneities on } \\
\text { fatigue-strength }\end{array}$ & MURAKAMI Y, ENDO M & IJFatigue & 1994 & 523 \\
\hline 12 & $\begin{array}{c}\text { A finite-volume-energy based approach to predict the } \\
\text { static and fatigue behavior of components with sharp } \\
\text { V-shaped notches }\end{array}$ & LAZZARIN P, ZAMBARDI R & IJFract & 2001 & 521 \\
\hline 13 & $\begin{array}{c}\text { Additive manufactured AlSi10Mg samples using } \\
\text { Selective Laser Melting (SLM): Microstructure, high } \\
\text { cycle fatigue, and fracture behavior }\end{array}$ & $\begin{array}{l}\text { BRANDL E, } \\
\text { HECKENBERGER U, } \\
\text { HOLZINGER V, et al }\end{array}$ & Mater. \& Des. & 2012 & 498 \\
\hline 14 & $\begin{array}{c}\text { Mechanisms of fatigue-crack propagation in ductile and } \\
\text { brittle solids }\end{array}$ & RITCHIE R O & IJFract & 1999 & 493 \\
\hline 15 & $\begin{array}{l}\text { Polarization fatigue in ferroelectric films: basic } \\
\text { experimental findings, phenomenological scenarios, } \\
\text { and microscopic features }\end{array}$ & $\begin{array}{l}\text { TAGANTSEV A K, } \\
\text { STOLICHNOV I, COLLA E L, } \\
\text { et al }\end{array}$ & J. App. Phys. & 2001 & 469 \\
\hline 16 & $\begin{array}{l}\text { Structural and functional fatigue of NiTi shape memory } \\
\text { alloys }\end{array}$ & $\begin{array}{l}\text { EGGELER G, HORNBOGEN } \\
\text { E, YAWNY A, et al }\end{array}$ & MSEA & 2004 & 460 \\
\hline 17 & $\begin{array}{c}\text { A crack opening stress equation for fatigue } \\
\text { crack-growth }\end{array}$ & NEWMAN J C & IJFract & 1984 & 458 \\
\hline 18 & $\begin{array}{c}\text { Geometrical effects in fatigue: a unifying theoretical } \\
\text { model }\end{array}$ & TAYLOR D & IJFatigue & 1999 & 417 \\
\hline 19 & $\begin{array}{l}\text { Fatigue and switching in ferroelectric memories- theory } \\
\text { and experiment }\end{array}$ & $\begin{array}{l}\text { DUIKER H M, BEALE P D, } \\
\text { SCOTT J F, et al }\end{array}$ & J. App. Phys. & 1990 & 413 \\
\hline 20 & $\begin{array}{l}\text { A model of extrusions and intrusions in fatigued } \\
\text { metals .1. point-defect production and the growth of } \\
\text { extrusions }\end{array}$ & $\begin{array}{l}\text { ESSMANN U, GOSELE U, } \\
\text { MUGHRABI H }\end{array}$ & $\begin{array}{l}\text { Philosophical } \\
\text { Magazine A }\end{array}$ & 1981 & 413 \\
\hline
\end{tabular}

注: 序号 1、2、4 和 5 为 Google Scholar 统计结果, 其余来自 Web of Science; 被引用数统计截止到 2020 年 11 月。

\section{3 关键研究领域分析}

\subsection{1 蠕变-疲劳领域}

蠕变-疲劳是复杂载荷模式的典型代表, 具有广 泛的工程需求。以 “Fatigue” 和 “Creep” 为检索词, 在 Web of Science 中检索 1970-2020 年期间发表论 文情况，结果如图 9 所示。可以看出，1970-1998 年期间, 论文数量逐年增加, 而 1999-2008 年期间, 论文数量处于低位, 随后论文数量基本保持在 70 篇/年左右。进一步以检索到的论文为对象, 分析了 蠕变-疲劳领域研究热点的变化。结果表明, 20 世纪 70 年代的工作聚焦于损伤模型的构建，而 20 世纪 80 年代的热点为裂纹扩展及预测、损伤机理与寿命 预测等方面。20 世纪 90 年代后的研究工作与高温 材料等有关, 例如氮化硅、 $\mathrm{SiC}$ 复合材料、铅锡焊 料及接头、高 $\mathrm{Cr}$ 耐热钢、镍基合金等分别成为热门
研究对象。近 10 年来, 人们更关注能量法损伤模型, 并在多轴加载行为、断裂参量和模型等方面开展了 较多的研究工作, 这与近年来中国、印度等国家大 力发展先进发电设备、航空发动机、燃气轮机等重 大装备有关。

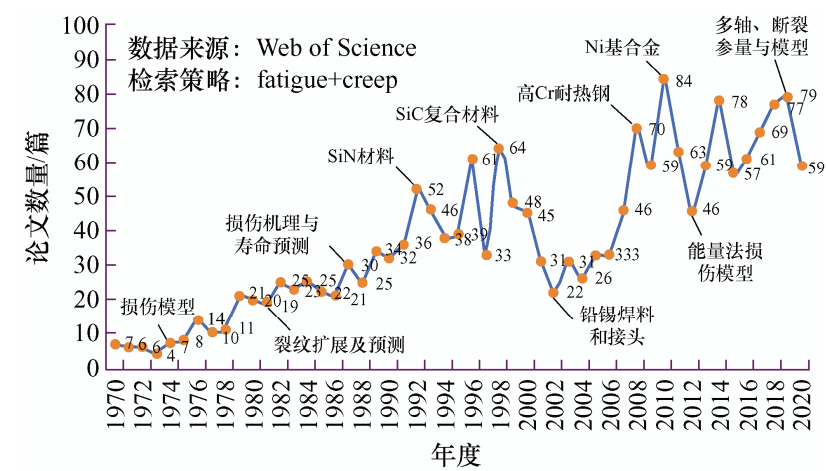

图 91970 - 2020 年间蠕变-疲劳研究论文发表数量及对象 
表 2 列出了 1970-2020 年间蠕变-疲劳研究方 向被引次数较多的论文。可以看出, 引用次数靠前 的论文主要发表于 20 世纪 70-80 年代, 论文报道 的内容主要涉及损伤模型、裂纹扩展、蠕变-疲劳氧化方面的损伤机制及寿命模型; 论文作者主要包
括法国 PINEAU 教授、美国 SEHITOGLU 教授等, 而论文主要的发表期刊包括 J. Eng. Mater. Tech.、 Mater. Sci. Eng.、Metal. Trans. A、IJFatigue 和 Acta Mater.等。

\section{表 $21970-2020$ 年间蠕变-疲劳研究方向被引次数较多的论文列表}

\begin{tabular}{|c|c|c|c|c|}
\hline 论文题目 & 作者 & 期刊 & 年度 & 引用数 \\
\hline Application of damage concepts to predict creep-fatigue failures & $\begin{array}{l}\text { LEMAITRE J; } \\
\text { PLUMTREE A }\end{array}$ & J. Eng. Mater. Tech. & 1979 & 179 \\
\hline $\begin{array}{l}\text { Accumulated creep strain and energy density based thermal fatigue } \\
\text { life prediction models for } \mathrm{SnAgCu} \text { solder joints }\end{array}$ & SYED A & $\begin{array}{l}54 \text { th Electronic Components } \\
\text { \& Technology Conference }\end{array}$ & 2004 & 175 \\
\hline $\begin{array}{c}\text { Thermomechanical fatigue, oxidation, and creep .1. Damage } \\
\text { mechanisms }\end{array}$ & $\begin{array}{l}\text { NEU R W; SEHITOGLU } \\
\text { H }\end{array}$ & Metal. Trans. A & 1989 & 140 \\
\hline Thermomechanical fatigue, oxidation, and creep .2 . Life prediction & $\begin{array}{l}\text { NEU R W, SEHITOGLU } \\
\text { H }\end{array}$ & Metal. Trans. A & 1989 & 129 \\
\hline $\begin{array}{l}\text { The effect of microstructure and environment on the crack growth } \\
\text { behavior of inconel } 718 \text { alloy at } 650{ }^{\circ} \mathrm{C} \text { under fatigue, creep and } \\
\text { combined loading }\end{array}$ & PEDRON J P, PINEAU A & Mater. Sci. Eng. & 1982 & 126 \\
\hline $\begin{array}{l}\text { Creep-fatigue-oxidation interactions in a } 9 \mathrm{Cr}-1 \text { Mo martensitic } \\
\text { steel. Part 1: Effect of tensile holding period on fatigue lifetime }\end{array}$ & $\begin{array}{l}\text { FOURNIER B, SAUZAY } \\
\text { M, PINEAU A }\end{array}$ & IJFatigue & 2008 & 102 \\
\hline $\begin{array}{c}\text { Evaluation of the strength and creep fatigue behavior of hot } \\
\text { isostatically pressed silicon-nitride }\end{array}$ & $\begin{array}{l}\text { FERBER M K, JENKINS } \\
\qquad \text { M G }\end{array}$ & JACS(ceramic) & 1992 & 100 \\
\hline $\begin{array}{l}\text { A generalized energy-based fatigue-creep damage parameter for } \\
\text { life prediction of turbine disk alloys }\end{array}$ & $\begin{array}{l}\text { ZHU Shunpeng, Huang } \\
\text { Hongzhong }\end{array}$ & EFM & 2012 & 99 \\
\hline $\begin{array}{c}\text { Constitutive relation and creep-fatigue life model for eutectic tin } \\
\text { lead solder }\end{array}$ & KNECHT S, FOX I R & $\begin{array}{l}\text { IEEE Trans. Comp. Hybrids } \\
\text { Manuf. Tech. }\end{array}$ & 1990 & 91 \\
\hline $\begin{array}{l}\text { A cohesive zone model for fatigue and creep-fatigue crack growth } \\
\text { in single crystal superalloys }\end{array}$ & $\begin{array}{l}\text { BOUVARD J L, } \\
\text { CHABOCHE J L, FEYEL } \\
\text { F, et al. }\end{array}$ & IJFatigue & 2009 & 91 \\
\hline
\end{tabular}

注：以上表格中被引次数的统计时间截至 2020 年 11 月。

\subsection{2 超高周疲劳领域}

超高周疲劳是对低周和高周疲劳研究的延伸, 是近 20 余年的新兴研究领域。以 “very high cycle fatigue" 、"ultralong cycle fatigue" 、 "gigacycle fatigue", "superlong life fatigue", "ultrahigh cycle fatigue” 为主题词, 通过 Web of Science 数据库共检 索到学术论文 581 篇(时间截至 2020 年 11 月)。从 图 10 所示的论文分布情况可见, 2006 年以后 SCI 论文数量处于较高水平, 2016 年的论文数量最多, 反映了这一领域逐年升温的研究态势。

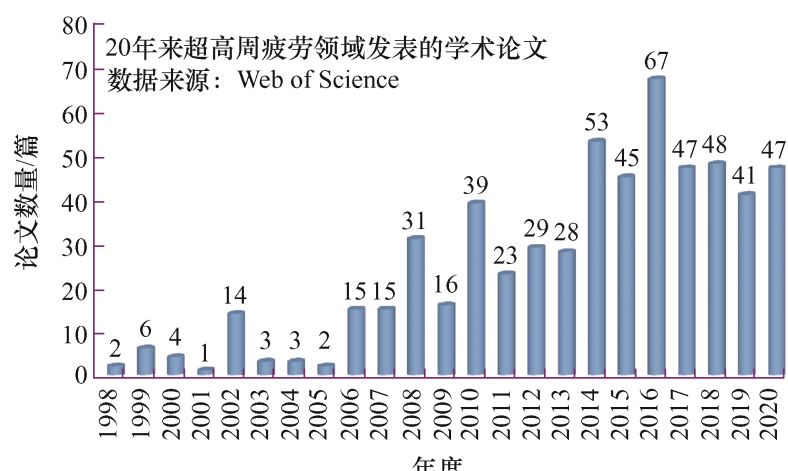

图 101998 - 2020 年超高周疲劳领域学术论文发表数量

\subsection{3 原位疲劳领域}

通过以 “in situ” 和 “fatigue” 为检索词, 在 Web of Science 中检索近 50 年来的论文发表情况,
结果如图 11 所示。可以看出, 20 世纪 70-80 年代 的论文数量很少, 90 年代后论文逐年增加, 至 2018 年论文数量达到 90 篇左右, 表明原位疲劳的研究逐 渐成为人们的研究热点。对发表论文进行分析, 可 以看出每一时期的研究热点与主题。图 11 中的文字 标注基本反映了原位疲劳领域关键技术的发展和进 步，表明原位疲劳试验是开展疲劳损伤机制或微细 观力学行为研究的重要手段。例如, 原位疲劳的研 究需求逐步与扫描电子显微镜 (Scanning electron microscopy, SEM)、透射电子显微镜(Transmission electron microscopy, TEM)、X 射线、中子散射、数 字图像相关法(Digital image correlation, DIC)、光学 显微镜以及同步辐射等技术结合起来。

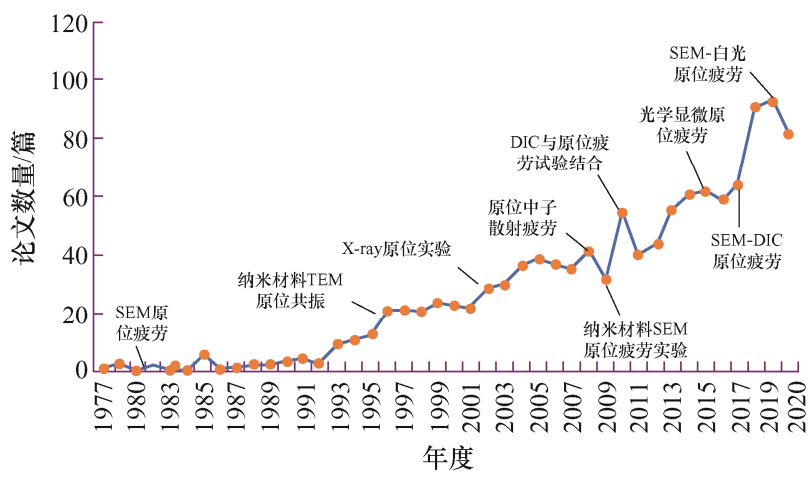

图 11 近 50 年来原位疲劳方向的论文发表情况及热点分析 


\subsection{4 有限元分析领域}

有限元分析是重要的研究工具, 其在疲劳领域 的应用能够反映技术发展的趋势。图 12 所示为 1970 -2020 年期间基于有限元分析方法开展疲劳研究 的论文分布情况。可以看出, 该领域论文发表数量 逐年增加, 表明疲劳分析计算技术逐渐成为人们的 研究工具, 尤其是近 10 年, 平均每年有 125 篇左右 论文发表。从论文报道的研究主题看, 20 世纪的研 究主要集中在应力分析、裂纹闭合和三维裂纹扩展 三个方面。进入 21 世纪以来, 有限元分析方法的进 步, 尤其是晶体塑性有限元和扩展有限元分析技术 的发展, 为复杂条件下的疲劳研究提供了重要支撑。 如图 12 中插图所示, 自 1992 年以来, 基于晶体塑 性方法的疲劳研究论文数量逐渐增加。扩展有限元 和晶体塑性有限元等方法的出现促进了人们对疲劳 裂纹扩展行为和多尺度疲劳损伤问题的研究, 为多 尺度、多场耦合条件下的疲劳破坏机制研究提供了 重要的工具。

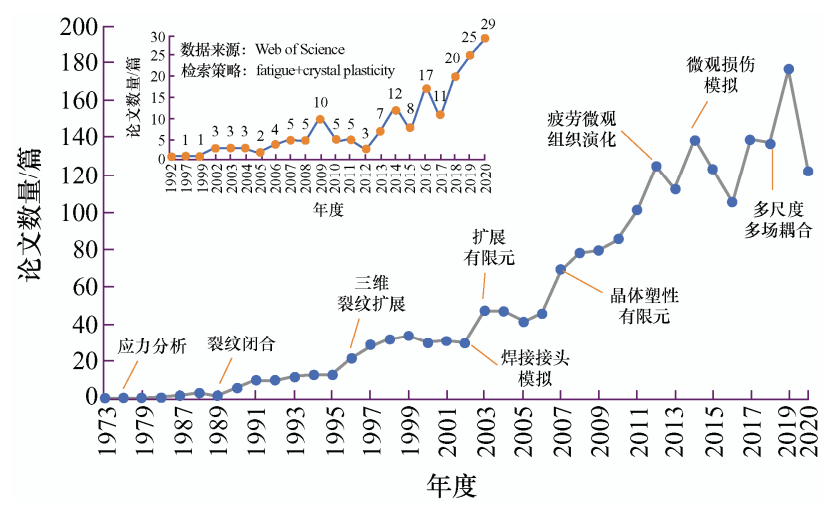

图 12 1970-2020 年期间疲劳有限元研究论文分布

\section{4 国内主要研究单位分析}

我国自 20 世纪 70 年代以来, 逐渐形成了一批
疲劳研究的队伍。图 13 为近 20 年中国发表疲劳领 域论文的研究团队统计情况, 共有 31 个单位的发文 数量超过 100 篇。进一步分析近 20 年的研究热点, 厘清发展变化趋势, 结果见表 3 。

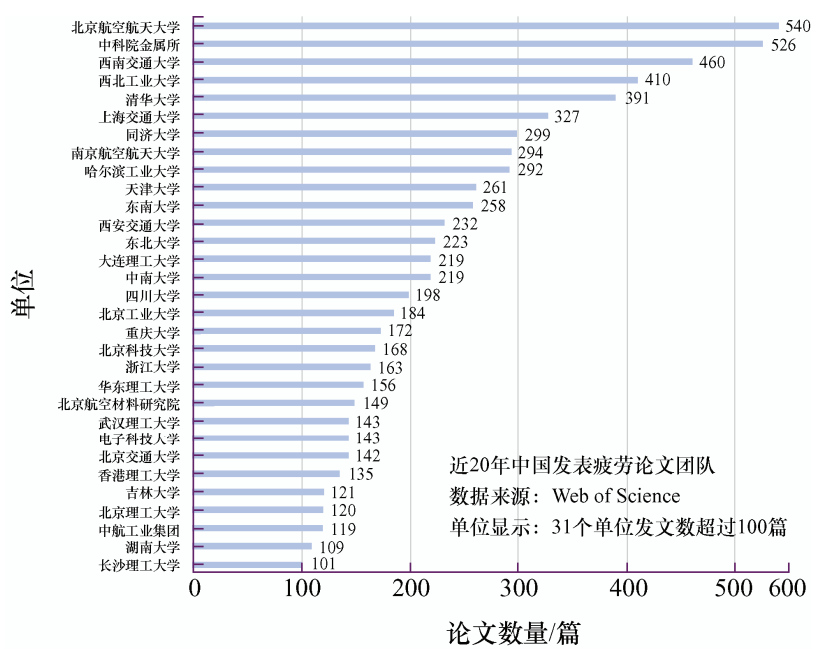

图 13 近 20 年(2001-2020)中国发表疲劳论文研究团队

由表 3 可见, 近 20 年间, 所选国内 7 家单位后 10 年的论文数量明显高于前 10 年, 这一方面与前 10 年的研究累积效应有关, 另一方面也表明疲劳研 究成为刚性需求。值得注意的是, 北京航空航天大 学的论文数量增幅最大, 成为后 10 年发表论文数量 最多的单位, 表明其在疲劳领域研究的强劲势头。 对于研究热点的变化, 中国科学院金属研究所的研 究热点受到纳米尺度断裂和增材制造的驱动, 清华 大学以新材料、原位疲劳测试及微观机制等研究为 特色, 华东理工大学更关注复杂载荷的疲劳及其损 伤监测等问题的研究。相关单位研究热点的变化, 一方面反映了国际学术前沿的动态, 另一方面也反 映了国内部分行业需求的变化。

表 3 国内相关单位近 20 年疲劳研究热点问题的比较

\begin{tabular}{|c|c|c|c|c|}
\hline \multirow{2}{*}{ 单位名称 } & \multicolumn{2}{|r|}{ 前 10 年 $(2001-2010)$} & \multicolumn{2}{|r|}{ 后 10 年(2011一2020) } \\
\hline & 论文数量 & 研究热点 & 论文数量 & 研究热点 \\
\hline 中国科学院金属研究所 & 237 & $\begin{array}{c}\text { 表面纳米化; 超高周疲劳; 铜薄膜/镁合金/ } \\
\text { 钛合金/金属玻璃 }\end{array}$ & 289 & $\begin{array}{c}\text { 梯度纳米化; 增材制造; 纳米孪晶铜; 孪晶界 } \\
\text { 断裂; 超高周疲劳 }\end{array}$ \\
\hline 北京航空航天大学 & 71 & $\begin{array}{c}\text { 热障涂层; 复合材料; 蠕变-疲劳; 金属 } \\
\text { 玻璃 }\end{array}$ & 469 & $\begin{array}{c}\text { 钛合金; 增材制造; 复合材料; 蠕变-疲劳; } \\
\text { 镍基合金; 纳米材料 }\end{array}$ \\
\hline 西南交通大学 & 102 & 超高周疲劳; 棘轮; 概率寿命与可靠性 & 358 & $\begin{array}{c}\mathrm{NiTi} \text { 合金; 高铁结构疲劳; 接触疲劳; 超高周 } \\
\text { 疲劳; 同步辐射 }\end{array}$ \\
\hline 清华大学 & 147 & 电极材料; 纳米材料; 铁电材料; 原位疲劳 & 244 & $\begin{array}{c}\text { 压电陶瓷; 超高周疲劳; 原位高温疲劳; 纳米 } \\
\text { 孪晶 }\end{array}$ \\
\hline 西北工业大学 & 107 & $\begin{array}{c}\text { 梀轮; 喷丸强化; 高低周复合; 超高周; 压 } \\
\text { 痕 }\end{array}$ & 303 & 焊接接头; 复合材料; NiTi 合金; 微动疲劳 \\
\hline 上海交通大学 & 66 & $\begin{array}{c}\text { 碳纳米管; 寿命预测; 镁合金; 蠕变-疲劳; } \\
\text { 涂层 }\end{array}$ & 261 & 镁合金; 焊接接头; 喷丸强化 \\
\hline 华东理工大学 & 33 & $\begin{array}{c}\text { 激光冲击; 表面纳米化; 涂层; 裂纹; 蠕变- } \\
\text { 疲劳 }\end{array}$ & 123 & $\begin{array}{c}\text { 蠕变-疲劳; 小裂纹; 超高周疲劳; 非线性 } \\
\text { 超声检测 }\end{array}$ \\
\hline
\end{tabular}




\section{5 中-美比较分析}

比较中国与美国在疲劳领域的差异, 可以 帮助我们找到短板, 实现超越。图 14 表示了 1970-2020 年间中国与美国发表的疲劳研究论 文数量比较结果。可以看出, 中国与美国发表论 文数量差异可分为三个阶段: 美国领先阶段(1970 - 2007 年)、中美相持阶段(2008-2010 年)和中国 赶超阶段(2011-2020 年)。在美国领先阶段, 美 国的论文数量明显多于中国, 中国自 1982 年论文 发表才逐渐起步; 在相持阶段, 中国与美国的论 文数量均在 300 篇/年左右; 在中国赶超阶段, 中 国发表的论文数量大幅度提升, 而美国的研究论 文数量呈波动性增长, 但增加缓慢。近 10 年, 中 国的㽻劳研究在体量上相对美国具有绝对优势, 中国赶超可能与日益增长的先进制造业发展需求 有关, 也表明中国在疲劳研究领域已是世界大国。 2020 年中、美发表的论文数量均略有下降, 可能 与新冠疫情对科研的影响有关。

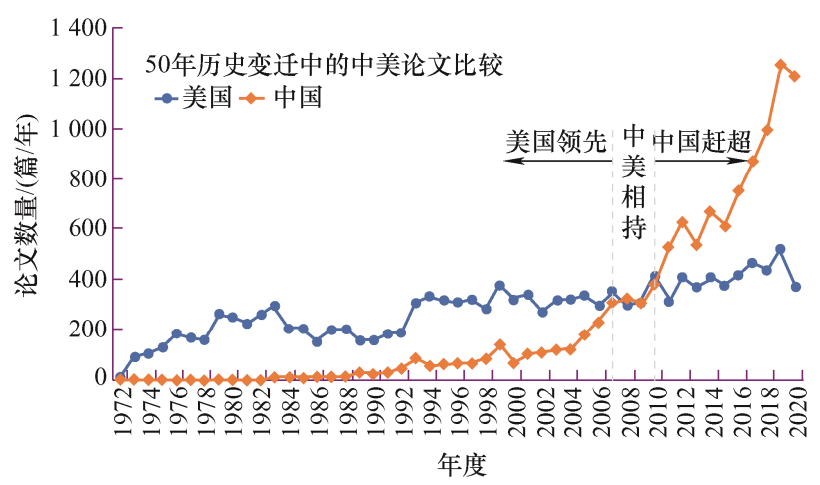

图 14 1970-2020 年间中国与美国发表论文数量比较

自 1998 年以来, 中国发表的论文数量稳步增 长。基于这方面的考虑, 对 1998-2020 年期间中国 与美国发表的论文, 分析较高被引次数论文的特点, 并采用关键词描述每 4 年间的研究热点, 如图 15 所示。从中国方面看, 疲劳研究主题经历着多轴疲 劳、棘轮、超高周疲劳和概率疲劳等过程, 热点研 究对象包括单晶铜、铁电材料、铜薄膜、复合材料、 岩石材料、梯度纳米晶、增材制造和隧道/桥梁等材 料与结构; 中国与美国共同关心的问题包括电极、 金属玻璃、镁合金、水凝胶和沥青混合物等材料的 疲劳, 以及疲劳损伤监测/检测等领域的研究。同时 可见, 超高周疲劳领域逐渐由共同热点转变为中国 热点, 疲劳损伤监测/检测领域由早期的美国热点转 变为中美共同热点, 而在增材制造、高熵合金等领 域, 美国先于中国开展研究。此外, 在碳纳米管、
燃料电池、镍基合金、石墨烯和镍钛合金等新材料 热点研究领域, 目前主要由美国主导, 中国仍需抓 紧赶上。

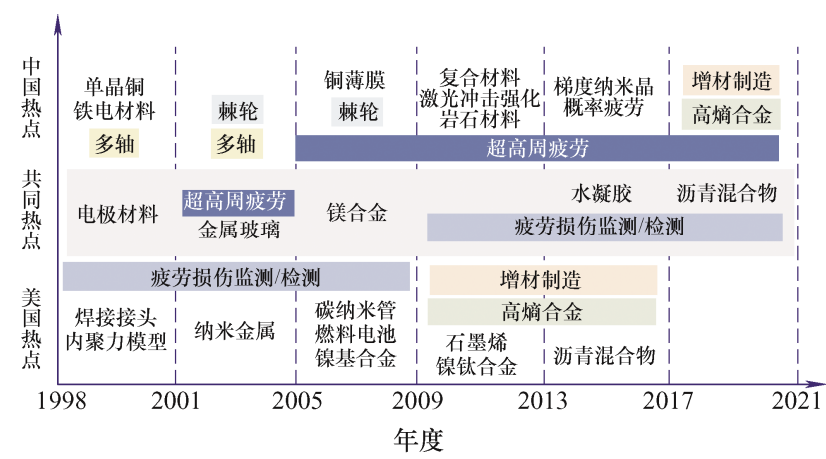

图 15 1998-2020 年间疲劳领域中国与美国研究热点变化

\section{6 疲劳研究的科学基金资助分析}

在国家自然科学基金学科分布方面, 与疲劳研 究相关的基金项目涉及机械工程、材料和力学等学 科, 本文着重分析疲劳研究在机械结构强度学(申请 代码 E0504)方向的资助情况。经统计, 自 1986 年 国家自然科学基金委员会成立迄今，E0504 已资助 面上、青年、地区等各类型项目共 761 项，资助金 额 36165.5 万元。其中, 与疲劳直接相关的项目共 计 229 项，资助金额 11292 万元，占比 $31 \%$ 。从项 目类型看，面上项目 105 项、青年基金 85 项、地区 基金 21 项、国际合作与交流 4 项、杰青 4 项、优青 3 项、海外青年合作基金 1 项、联合基金 3 项、重 点项目 3 项和重大研究计划项目 1 项, 多类型项目 的资助表明疲劳研究领域相当活跃。

对 1993-2020 年间疲劳研究获科学基金资助 情况进行统计, 结果如图 16 所示。可以看出, 在 $1993-2000$ 年、2001-2010 年和 2011-2020 年三 个时间段，科学基金的资助数量呈现出较低水平、 稳步提升和快速增加的特点。1993-2000 年间共资 助 13 项, 项目主题涉及疲劳监测技术、多轴疲劳和

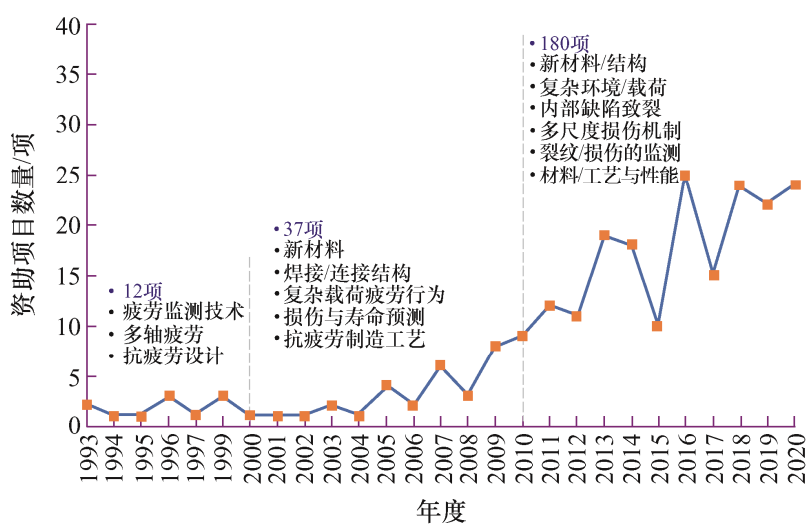

图 16 1993-2020 年间疲劳研究获科学基金资助情况 
抗疲劳设计等; 2001-2010 年间资助项目有所增 加, 共资助 37 项, 项目主题涉及新材料、焊接/连 接结构、复杂载荷疲劳行为、损伤与寿命预测、抗 疲劳制造工艺等, 体现了增长的工程需求对疲劳 研究的推动作用; 2011-2020 年间资助数量快速增 加, 达到了 180 项, 项目研究主题涉及新材料/结构、 复杂环境/载荷、内部缺陷致裂、多尺度损伤机制、 裂纹/损伤的监测、材料/工艺与性能等多个方面, 很 大程度上受到知识获取的驱动, 体现了人们对疲劳 理论与方法研究在广度和深度方面的拓展, 并逐渐 与制造工艺相融合。

对基金资助项目的研究主题与前述国际研究热 点进行比较, 可以看出部分受资助项目的研究对象、 所采用的方法和科学问题具有明显的创新性。例如, 1993 年批准的基金面上项目 “金属内部及表面疲劳 极限研究”, 当时处于国际学术前沿, 至今也是人们 开展超高周疲劳研究需要考虑的科学问题; 1994 年 批准的青年基金 “运用神经网络的疲劳过程实时监 测方法的研究”, 对于近年来装备的损伤监测与智能 运维技术研究具有引领性。因此, 自然科学基金资 助的疲劳领域科研项目创新性明显, 并具有较好的 前瞻性和引领性。科学基金已成为引导科学家探索 科学前沿、潜心开展基础研究、提升学科国际影响 力的重要渠道。

\section{7 从热点看趋势}

近 50 年疲劳领域的研究热点分析清楚表明, 前期研究侧重于疲劳基础理论框架的发展, 诸如 多向应力、短裂纹、裂纹闭合和复合材料的研究 丰富了疲劳的早期理论, 形成了人们熟知的疲劳 理论框架。这一阶段的疲劳研究受到工程需求的 推动, 尤其是工程事故调查与取证的驱动, 其主 要目标是满足新型工业装备的设计与制造需求, 进而提高工程结构的服役安全性。进入 21 世纪以 来, 疲劳研究聚焦于非传统材料和非传统制造工 艺等新兴领域, 诸如高熵合金、增材制造等带来 的新疲劳问题扩大了疲劳研究对象, 丰富了疲劳 理论与方法。原位试验技术和有限元分析方法的 进步为深入分析疲劳行为和多尺度损伤机制提供 了很好的工具, 使疲劳这一古老的问题焕发出新 的生机与力量, 也使疲劳领域呈现出了 “永远有 疲劳问题研究” 且 “永不疲劳的研究” 的景象, 这一阶段的研究以知识驱动作为主要特点, 人们 期待着提供更多的科学证据以解释传统现象, 或 者拓展现有疲劳理论至新的未知领域。因此, 知 识驱动型的疲劳研究将会成为新阶段主流趋势。

\section{3 主要研究进展}

分析百年来疲劳领域的论文发表情况, 有助于 从研究热点的变化中发现新趋势, 而掌握疲劳领域 的具体进展有助于明确现状与问题, 进而最终提出 新趋势下的研究思路。

\section{1 里程碑式工作}

自 “疲劳”一词正式被提出以来, 人们围绕疲 劳问题开展了诸多研究, 很多标志性工作奠定了疲 劳的重要理论基础, 具有里程碑式意义 ${ }^{[17]}$ 。图 17 表示了 1854-2020 年期间疲劳领域里程碑式的工 作, 图中年份特指相关研究成果发表见刊的时间。 可以看出, 从 1870 年德国工程师 WÖHLER 提出 $S-N$ 曲线和疲劳极限概念开始 ${ }^{[18-19]}, 19$ 世纪末另一具有 重要意义的工作是包辛格效应的提出 ${ }^{[20]} ; 20$ 世纪前 半叶的重要工作包括 BASQUIN 建立应力-寿命关 系 ${ }^{[21]}$ 、PALMGREN-MINER 提出的损伤累积准 则 ${ }^{[22-23]}$, 以及 MANSON-COFFIN 关系的提出 ${ }^{[24-25]}$ 。 20 世纪 60 年代后, 随着微观分析手段的进步和断 裂力学的发展, 疲劳领域涌现出大量具有重要意义 的工作。FORSYTH ${ }^{[26]}$ 与 RYDER $^{[27]}$ 利用微观电镜技 术观察到了疲劳断口存在辉纹; NEUBER ${ }^{[28]}$ 建立了 较为成熟的缺口应力理论, 为深入认识疲劳极限和 结构设计提供了基础; PARIS 等 ${ }^{[13]}$ 对疲劳裂纹扩展 行为进行描述, 提出了裂纹扩展速率与应力强度因 子范围的幂函数关系, 即 PARIS 公式; MATSUISHI 与 $\mathrm{ENDO}^{[29]}$ 提出雨流计数法, 解决了变幅载荷下的 循环数计算, 为结构寿命估算提供了依据。

20 世纪 70 年代, ELBER 发现裂纹闭合现象 ${ }^{[14]}$, 成为伴随裂纹扩展过程的一个重要特征, 至今仍是 研究热点; BROWN 与 MILLER ${ }^{[30]}$ 建立了多轴疲劳 强度与寿命关系; MILLER ${ }^{[31]}$ LANKFORD ${ }^{[32]}$ 、 SURESH 等 ${ }^{[33}$ 和 RITCHIE 等 ${ }^{[34]}$ 系统研究小 (短)裂 纹扩展行为, 提出了疲劳裂纹扩展的短裂纹效应; RITCHIE 与 SURESH 对近门槛值区疲劳裂纹扩展 行为及断裂机理进行研究 ${ }^{[35-37]}$, 为疲劳门槛值测 试和损伤容限设计提供了理论基础。进入 20 世纪 80 年代, 我国科学家高镇同、欧进萍和阎楚良在 疲劳统计学和概率疲劳方面的工作为疲劳的工程 应用提供了基础 ${ }^{[38-40]}$ 。20 世纪 90 年代后期, 超 高周疲劳逐渐成为研究热点, BATHIAS ${ }^{[41]}$ 和 MURAKAMI 等 ${ }^{[42]}$ 较早开展了相关研究工作。进 入 21 世纪, MCDOWELL 和 DUNNE ${ }^{[43]}$ 提出微观 组织敏感的疲劳模拟方法, 至今仍是本领域的热门 研究领域。 


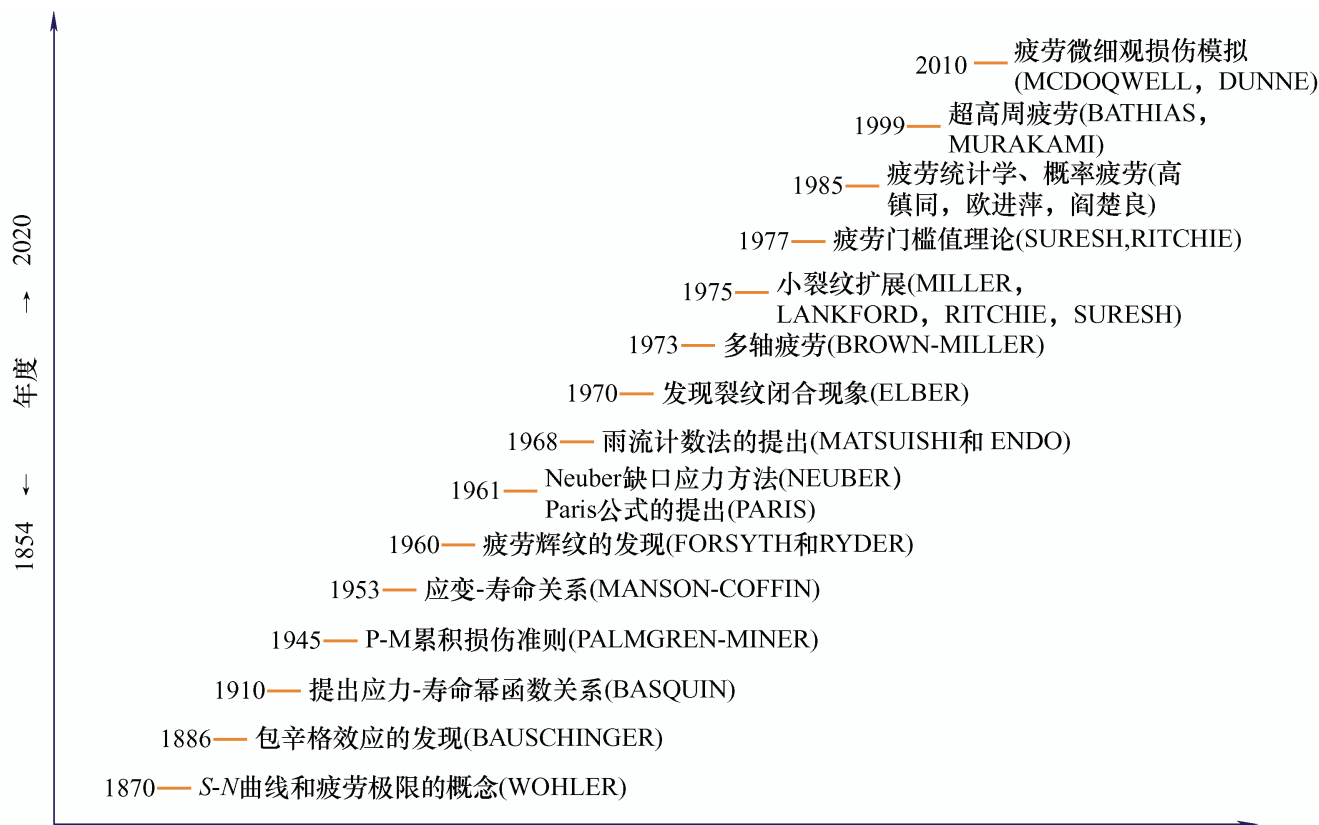

重要研究进展

图 17 1854-2020 年期间疲劳领域的里程碑工作

\section{2 基于 $S-N$ 曲线的欧洲疲劳设计理论体系}

WÖHLER ${ }^{[18]}$ 对铁路车轴钢的疲劳试验研究, 研 发了旋转弯曲疲劳试验装置, 并于 1870 年首次提出 了 $S-N$ 曲线及疲劳极限概念, 被工程领域沿用至 今 ${ }^{[19]}$ 。在 $S-N$ 曲线基础上, 人们分析发现循环应力 幅比最大应力更重要, GOODMAN、GERBER、 SODERBERG 等相继考虑平均应力效应 ${ }^{[44]}$, 建立的 疲劳应力幅-平均应力关系曲线沿用至今 ${ }^{[1]}$, BASQUIN $^{[21]}$ 构建了应力幅与疲劳寿命的关系式。应 力-寿命关系应用到变幅载荷条件需要疲劳损伤的 计算方法, 而 PALMGREEN 和 MINER 提出的线性 累积损伤提供了重要的准则 ${ }^{[22-23]}$, 至今成为疲劳寿 命计算的主要路线, 而 MATSUISHI 与 $\mathrm{ENDO}^{[29]}$ 提 出的雨流计数法为变幅载荷计算提供了准确的方 法。基于 $S-N$ 曲线发展起来的疲劳损伤与寿命计算 方法, 成为欧洲国家开展结构抗疲劳设计的理论基 础, 在铁路、航空等领域发挥着重要作用。

\section{3 基于应变-寿命曲线的美国疲劳设计理论体系}

20 世纪 50 年代的多起疲劳事故使人们关注到 缺口疲劳行为, 而疲劳试验技术的发展使高应力水 平下的疲劳测试成为可能。20 世纪 50 年代, 美国 BALDWIN 公司的 HEAD 和 ANON 研制了基于液 压伺服系统的疲劳试验机, 为精准控制应变提供了 技术基础 ${ }^{[45]}$; 美国 NASA 研究员 MANSON(1953 年)和 COFFIN(1954 年)开展应变控制的高应力幅疲 劳试验, 发现塑性应变与疲劳寿命的函数关系 ${ }^{[24-25]}$ 。 在塑性应变与疲劳寿命关系基础上, 围绕缺口疲劳
问题, 人们相继提出了描述缺口应力应变的 NEUBER 规则 ${ }^{[28]}$ 和 SMITH-WATSON-TOPPER 缺口 损伤参量 ${ }^{[46]}$, BROWN 与 MILLER 发现多轴疲劳的 最大损伤平面 ${ }^{[30]}$, 而 FATEMI 与 $\mathrm{SOCIE}^{[47]}$ 提出了多 轴疲劳临界面法。应变-寿命关系以及缺口疲劳理论 发展成为美国疲劳设计的基础理论, 在航空航天等 结构抗疲劳设计中发挥着重要作用。

\section{4 我国结构疲劳研究的主要进展}

20 世纪 70 年代开始, 国内对疲劳的研究逐渐 重视起来, 70 年代初期的工作主要围绕疲劳试验机 的设计与制造进行。70 年代后期, 颜鸣臬 ${ }^{[9,48-49]}$ 系 统综述了疲劳裂纹扩展规律及微观机制的进展, 师 昌绪等报道了蠕变与低周疲劳的交互作用行为 ${ }^{[50]}$, 徐影 ${ }^{[51]}$ 提出了疲劳强度可靠性设计的重要性, 张康 达 ${ }^{[22]}$ 分析了压力容器疲劳设计的 ASME 标准, 中国 航发北京航空材料研究院(原 621 研究所)讨论了喷 丸强化对高温疲劳的影响 ${ }^{[3]}$, 国内一些单位翻译整 理了国外的研究成果 ${ }^{[54-55]}$ 。进入 80 年代后, 国内对 疲劳的研究逐渐兴起, 郑修麟 ${ }^{[56]}$ 提出了预测裂纹萌 生寿命的局部应力-应变法, 高镇同等 ${ }^{[38}$ 提出了疲劳 强度的概率分析方法, 阎楚良等 ${ }^{[39,57]}$ 提出了载荷谱 的编制方法。90 年代, 谢里阳等 ${ }^{[58]}$ 发展了概率累积 损伤准则, 姚枚等提出了内部疲劳极限的概念 ${ }^{[59-60]}$, 陈旭等 ${ }^{[61]}$ 研究了非比例加载的多轴低周疲劳, 欧进 萍等 ${ }^{[40]}$ 发展了概率疲劳累积损伤方法, 姚卫星 等 ${ }^{[62-63]}$ 提出了结构抗疲劳设计的应力场强方法, 尚 德广等 ${ }^{[64]}$ 建立了多轴疲劳损伤模型。进入 21 世纪 
后, 疲劳研究领域得到扩大, 由轨道交通、压力容 器和航空航天等领域进一步拓展到土木、矿业、汽 车、发电、船舶和新材料等领域 ${ }^{[65-72]}$ 。疲劳理论与 方法得到进一步发展, 如赵永翔 ${ }^{[73]}$ 提出了应变疲劳可 靠性分析方法, 王清远、薛红前、轩福贞、王弘、李 守新和洪友士等相继开展了超高周疲劳研究 ${ }^{[74-79]}$, 赵 振业 ${ }^{[80]}$ 提出了抗疲劳制造技术, 吴学仁等 ${ }^{[81]}$ 提出了基 于小裂纹理论的疲劳全寿命预测方法, 概率疲劳寿命 预测成为解决结构疲劳的重要方法 ${ }^{[82-85]}$ 。

\section{5 疲劳裂纹扩展机制及理论描述}

在裂纹扩展方面, 图 18 表示了连续介质框架下 疲劳裂纹扩展行为的统一描述。可以看出, 疲劳裂 纹的扩展过程中, 在裂纹长度增加的同时, 对应的 物理机制发生着重要转变, 大体上可以分为不连续 机制、连续性机制和快速断裂主导的三个阶段。在 不连续机制主导阶段, 疲劳裂纹以纳米尺度、物理 短裂纹和近门槛值区裂纹呈现, 此时裂纹短、裂纹 扩展不连续, 疲劳裂纹扩展速率与应力强度因子范
围 $\Delta K$ 的规律不明显、随机性大。人们研究发现, 疲 劳裂纹萌生及早期扩展寿命约占到疲劳总寿命的 $70 \% \sim 80 \%$, 甚至更多。PEARSON ${ }^{[86]}$ 对短裂纹的研 究发现短裂纹扩展速率可以快于长裂纹, 即 “短裂 纹效应”; KITAGAWA-TAKAHASHI 图的提出完整 描述了短-长裂纹的转变机制 ${ }^{[87]}$, 而 EL HADDAD、 TOPPER 和 SMITH 等 ${ }^{[88-89]}$ 推导了短-长裂纹转变的 临界裂纹长度公式; SURESH、RITCHIE 和 LIAW 等 ${ }^{[36-37,90]}$ 系统阐明了裂纹闭合机制和理论模型;

NAVARRO 与 RIOS 等 ${ }^{[1-92]}$ 提出了短-长裂纹统一扩 展模型, 解决了裂纹随机扩展的理论描述问题; NEWMAN 和 WU 等 ${ }^{[93-94]}$ 提出了基于短裂纹理论的 寿命预测方法; ZHU 和 XUAN 等 ${ }^{[95-96]}$ 建立了近门 槛值区疲劳裂纹扩展的统一模型。在连续扩展机制 阶段, 疲劳裂纹扩展速率与 $\Delta K$ 呈现明显的线性关 系, 疲劳裂纹扩展基本可用 PARIS 公式进行描述。 在快速断裂区, 疲劳裂纹扩展速率增加很快, 占疲 劳总寿命分数较少。

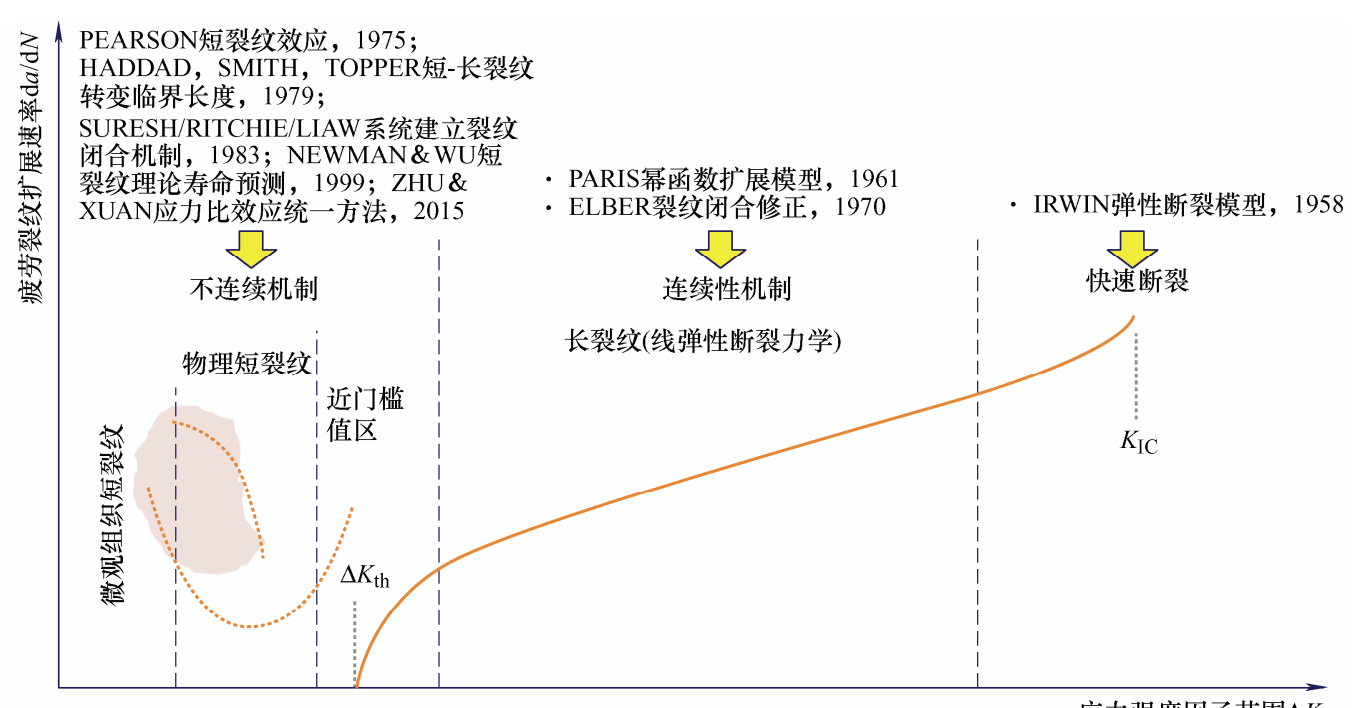

应力强度因子范围 $\Delta K$

图 18 连续介质力学框架下的疲劳裂纹扩展机制及理论描述

\section{6 蠕变-疲劳交互效应}

蠕变-疲劳交互效应的提出与人们对更高温度 的利用密切相关。1951 年, 美国 Argonne 国家实验 室建造首台快中子增值核反应堆(EBRI-1), 提出蠕 变-疲劳交互作用是潜在破坏问题。1963 年 ASME 标准 Code Case1331 首次提出线性累积损伤模式计 算作为蠕变-疲劳交互设计准则。随后, 蠕变-疲劳 交互作用的潜在破坏性进一步得到了两个失效事故 的证实, 即 1974 年美国 TVA Gallatin 热电厂 2 号机 组转子蠕变-疲劳断裂失效事故和 1979 年美国 Sabine 热电厂再热管发生的蠕变-疲劳失效事故。

围绕蠕变-疲劳问题, 人们在损伤累积与寿命预
测方面取得了重要进展。1963 年, ASME Code Case N-47 提出采用 MINER 线性疲劳损伤和 ROBINSON 蠕变损伤叠加的路线，这一技术路线被工程沿用至 今; 1971 年, MANSON 和 HALFORD 等 ${ }^{[97]}$ 提出应 变范围划分模型 SRP, 被 NASA 采用; 1975 年, LEMAITRE 和 CHABOCHE ${ }^{[98]}$ 以线性损伤累积律为 基础, 建立损伤理论模型; 1976 年, COFFIN 等 ${ }^{[99]}$ 提出了基于 MANSON-COFFIN 公式的频率修正模 型; 1980 年, HALES 等 ${ }^{[100]}$ 提出了韧性耗竭模型, 被 R5 采用; 2013 年, TAKAHASHI 等 ${ }^{[101]}$ 正式发表 了应变能密度耗竭模型的工作。

对蠕变及蠕变-疲劳裂纹扩展的研究是重要的 
方面。1976 年, LANDES 与 BEGLEY ${ }^{[102]}$ 率先建立 稳态蠕变断裂参量; 1981 年, BASSANI 和 MCCLINTOCK $^{[103]}$ 提出过渡蠕变范畴的参量 $C(t)$; 1986 年, SAXENA ${ }^{[104]}$ 提出小范围和过渡蠕变范畴 的参量 $C_{\mathrm{t}} ; 1990$ 年, YOON 与 SAXENA 等 ${ }^{[105]}$ 建立 包含蠕变影响的蠕变-疲劳裂纹扩展模型, 被 EPRI 采用; 2004 年, XUAN 和 TU 等 ${ }^{[106]}$ 提出了非均匀 材料的参量 $C_{\mathrm{t}} ; 2009$ 年, XUAN 和 $\mathrm{TU}$ 等 ${ }^{[107]}$ 建立 了多裂纹干涉与合并的判据与模型。此外, 人们基 于蠕变强度理论对高温设备进行安全评价, 1975 年 DOWLING 和 TOWNLEY ${ }^{[108]}$ 提出的延性断裂与塑 性失效评价方法, 被轩福贞和涂善东于 2006 年发展 成为包括蠕变损伤、延性断裂和塑性失效等多失效 模式的协同评价方法 ${ }^{[109]}$ 。人们对蠕变-疲劳交互效 应的微观机制方面, HALES ${ }^{[110-111]}$ 系统阐明微观机 制的载荷依赖性, 而产生蠕变-疲劳交互的应力区间 问题仍不明确, 2017 年 GONG 和 XUAN ${ }^{[112]}$ 阐明蠕 变-疲劳交互效应下的缺口强化和弱化机制。

\section{7 腐蚀疲劳模型}

疲劳与腐蚀环境的结合促成了腐蚀疲劳研究, 人们对腐蚀疲劳的研究仍起源于相关的事故, 尤其 是与材料与结构的季节性开裂密切相关。例如, 早 在 1860 年左右, 英军在印度最早报道发现大量弹壳 黄铜发生了 “季节开裂” (应力腐蚀), 而最早的腐 蚀疲劳事故报道于第一次世界大战期间, 当时英国 海军扫雷器引线破裂, 其后发现大量轮轴、锅炉等 设备的腐蚀疲劳事故 ${ }^{[113]} ; 1988$ 年, 美国 Aloha 波 音 737 飞机发生事故, 分析表明沿海飞行导致的含 盐湿度致腐蚀疲劳是重要原因, 也引起人们对老化 的航空结构完整性问题的重视 ${ }^{[114]}$ 。

人们关于腐蚀疲劳的研究最早可追溯到 1917 年 HAIGH 发表腐蚀疲劳的试验报告, 随后 1926 年, MCADAM 和 LEHMANN 发表腐蚀疲劳方面的论 文, 并提出 “腐蚀-疲劳” 概念, 成为 20 世纪冶金 学的重大进展 ${ }^{[113]}$ 。1944 年, 由 ASTM、AIME 发起 第一个关于 “金属应力腐蚀开裂研讨会” 在美国费 城召开, 主要讨论黄铜等腐蚀开裂 ${ }^{[115]}$; 60 年代, LECKIE $^{[116]}$ 首先采用电位法研究裂纹生长速率与施 加电位的关系, 成为当前腐蚀疲劳裂纹生长规律研 究的主流方法, WEI 等 ${ }^{[117-119]}$ 提出腐蚀疲劳裂纹扩 展的叠加模型, 未考虑机械和环境成分影响; 1977 年, AUSTIN 和 WALKER ${ }^{[120]}$ 认为机械、化学作用 是竞争而不是叠加的, 腐蚀疲劳裂纹生长速率取决 于两个因素谁占优势。进入 21 世纪, 腐蚀疲劳相关 的研究更多聚焦于微纳观尺度的机理研究, 以从根
本上阐明腐蚀疲劳破坏的微观机制 ${ }^{[121]}$ 。

\section{8 超高周疲劳行为}

近 20 年来, 超高周疲劳行为的研究是疲劳界的 热点, 其根本原因在于人们对传统疲劳极限是否存 在产生的疑问。1984 年, 日本学者 NAITO 等 ${ }^{[122]}$ 报道了 $50 \mathrm{~Hz}$ 下超过 $10^{8}$ 次应力循环的旋转弯曲疲 劳试验结果, 发现应力低于传统疲劳极限时失效仍 会发生。传统疲劳极限是否存在的问题, 引起了疲 劳界的广泛兴趣 ${ }^{[123]}$ 。2005 年, CHANDRAN 等 ${ }^{[124-125]}$ 研究表明超高周疲劳寿命受材料微缺陷和微结构主 导, 内部或亚表面为起裂位置, 应力-循环次数表现 为双 $S-N$ 曲线现象。另一方面, 超高周疲劳增长的 研究兴趣也与工程结构的长寿命服役需求有 关 $^{[76,126-127]}$ 。例如, 发动机部件、汽车承力运动部 件、铁路轮轴和轨道、飞机、海岸结构、桥梁和特 殊医疗设备等, 往往承受高频低应力幅循环载荷作 用, 实际的疲劳寿命已经超过了 $10^{7}$ 周次, 对这类 构件的抗疲劳设计迫切需要获得更长寿命条件下疲 劳破坏行为的科学认知。

人们特别关注超高周疲劳内部微缺陷处的裂纹 萌生与扩展机制 ${ }^{[128-129]}$, 至今已提出了多个缺陷致 裂机理模型。例如, 日本学者 MURAKAMI 等 ${ }^{[22]}$ 提出氢元素 $\left(\mathrm{H}_{2} 、 \mathrm{H} 、 \mathrm{H}^{+}\right)$致裂机制, 建立了夹杂物与 寿命的关系; WANG 等 ${ }^{[130]}$ 提出疲劳裂纹萌生与扩 展寿命模型; SHIOZAWA 等 ${ }^{[131]}$ 提出碳化物脱粘模 型; SAKAI 等 ${ }^{[132-134]}$ 提出了细晶区细化脱粘模型; GRAD 等 ${ }^{[135]}$ 提出晶粒细化模型; CHAI 等 ${ }^{[136-137]}$ 认 为缺陷致裂与局部塑性变形有关; HONG 等 ${ }^{[138-139]}$ 提出大数循环压缩模型; ZHU 和 XUAN 等 ${ }^{[140-141]}$ 提出了马氏体断裂形成位错胞结构和塑性能量累积 模型。

\section{9 疲劳试验技术}

疲劳试验技术的发展与进步是疲劳研究的重要 支撑。对于疲劳的研究, 试验技术主要包括疲劳试 验机和微观分析技术的发展。疲劳试验机是人们获 取疲劳数据的基本工具。1870 年, WÖHLER ${ }^{[19]}$ 报 道了旋转疲劳试验机, 使开展试样的疲劳试验成为 可能; 1938 年, 瑞士 Amsler 公司 RUSSENBERGE 等报道了高频疲劳试验机 ${ }^{[142]} ; 1950$ 年, HEAD 和 ANON 发明了液压伺服疲劳试验机 ${ }^{[45]}$; 此外, 早在 1950 年 MASON 等报道了可在 $20 \mathrm{kHz}$ 下振动的超 声疲劳试验机，至 1959 年 NEPPIRAS 首次报道用 超声疲劳试验机测量得到的 $S-N$ 曲线 ${ }^{[143]}$, 近 20 年 来超声疲劳试验机重新受到重视, 成为热门工具, 被用于研究多种材料的超高周疲劳行为 ${ }^{[144]}$ 。 
人们观察物质最初使用光学显微镜, 20 世纪早 期, EWING 和 HUMPHREY ${ }^{[145]}$ 报道微裂纹形成过 程中的表面滑移形貌, GOUGH ${ }^{[6]}$ 研究了弯曲与扭转 共同作用下的疲劳机理。为突破光学显微镜在放大 倍数和分辨率的局限性, 1932 年, 克诺尔和鲁斯卡 发明透射电子显微镜, 至 1970 年, 芝加哥大学的克 鲁发明了场发射枪, 场发射透射电子显微镜出现; 1938 年, ARDENNE 发明了扫描电子显微镜, 1965 年第一部商用 SEM 在英国剑桥出现, 日本电子于 1966 年生产第一台商用 $\mathrm{SEM}^{[146]}$ 。电子显微技术的 发展为人们研究疲劳微观机制提供了高精度的工 具 ${ }^{[147]}$ 。此外, 人们也开发并利用同步辐射技术研究 材料内部的损伤演化行为, 2000 年, 法、日科学家 分别报道了基于该技术的疲劳研究结果 ${ }^{[148-149]}$, 该 技术成为近 20 年来热门的研究手段 ${ }^{[150-152]}$ 。图 19 表示了疲劳试验机和微观分析技术的进步。

除了对瘦劳后试样的损伤与断裂机制进行观测 外, 能够实时观测疲劳损伤过程的试验方法, 已成 为讨论疲劳微观机制的主流手段。1969 年, DINGLEY $^{[153]}$ 首次报道了块体材料在 SEM 内的原 位试验装置; 1979 年, VEHOFF 与 NEUMANN ${ }^{[154]}$
报道了块体材料在 SEM 的原位疲劳研究结果; 1999 年, PONCHARAL 等 ${ }^{[155]}$ 开展了纳米材料在 TEM 中 的原位共振疲劳试验, 同年 MORANO 等 ${ }^{[156]}$ 利用 X-ray 原位疲劳装置观测疲劳裂纹的形貌, 分析裂纹 闭合效应; 2000 年以来, 人们逐渐开始使用中子散 射与同步辐射技术研究结构残余应力测量 ${ }^{[157]}$ 、疲劳 裂尖场测定 ${ }^{[158-159]}$ 等; 2009 年, VANLANDUIT 等 ${ }^{[160]}$ 将 DIC 技术用于原位分析疲劳裂纹扩展及应力强度 因子; 2012 年, ZYBELL 等 ${ }^{[161]}$ 采用光学显微原位 疲劳技术，分析疲劳裂纹扩展时的过载效应; 2015 年, LU 和 ZHU 等 ${ }^{[162-163]}$ 利用 DIC 技术表征疲劳裂 尖场, 提出了疲劳裂纹扩展的临界应变准则; 2018 年, YANG 等 ${ }^{[164-165]}$ 将 DIC 技术用于研究描述疲劳 裂纹扩展的 CJP 模型; 2020 年, YIN 等 ${ }^{[166]}$ 发展了 高温 SEM-DIC 原位疲劳技术，研究超强合金的高 温疲劳裂纹扩展行为, CHEN 等 ${ }^{[167]}$ 将同步辐射技术 与原位 SEM/DIC 结合起来分析疲劳裂纹过载机制。 可以看出, 光学显微镜、电子显微镜、先进同步辐 射以及中子散射等技术提供了不同尺度和精度下疲 劳裂纹观测手段, 对系统揭示疲劳破坏的微观机制 和规律具有重要价值。

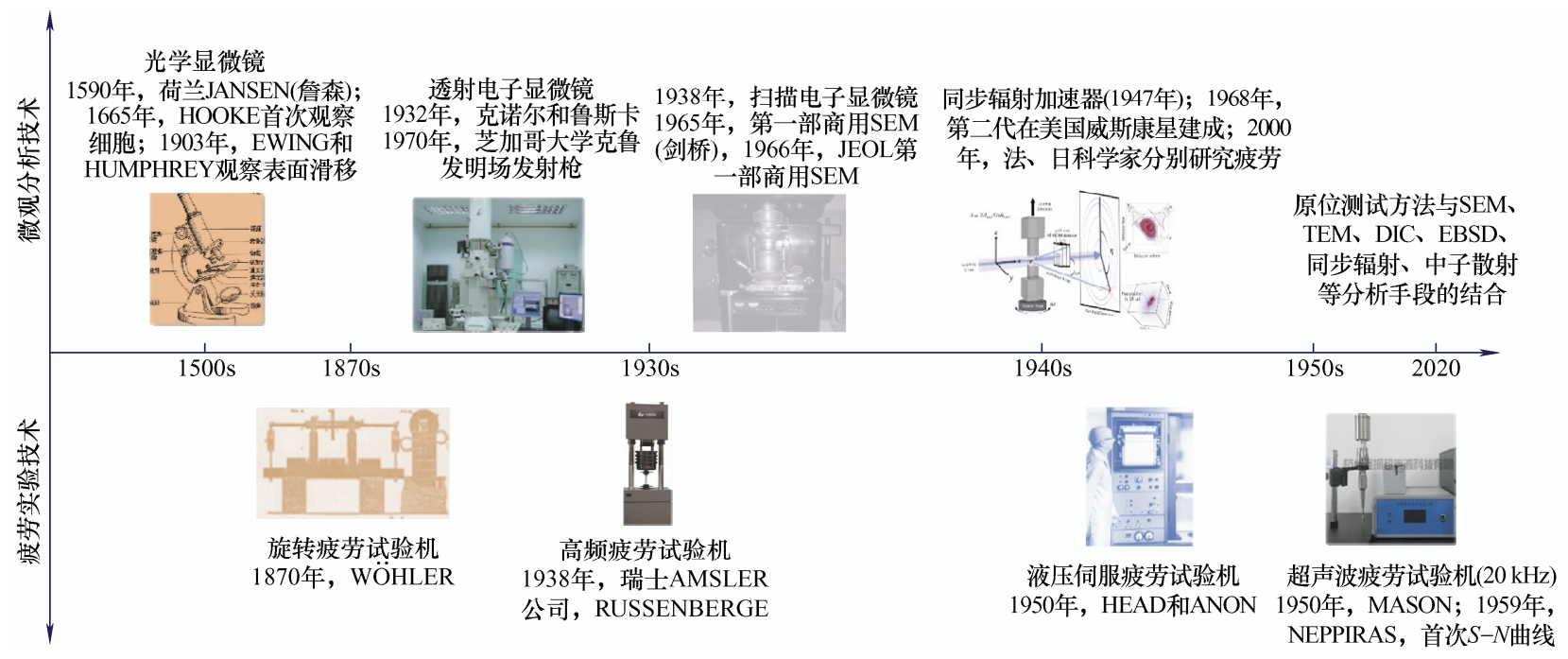

图 19 疲劳试验机及微观分析技术的发展与进步

\subsection{0 疲劳分析计算技术}

疲劳分析计算技术可为疲劳试验结果提供佐 证, 并能提供试验无法提供的科学认知。实现疲劳 分析需要具备计算能力和分析方法。有限元分析方 法的发展与计算能力的提升支撑了疲劳分析, 目前, 人们基于疲劳分析工具已经获得了诸多重要进展。 1973 年, 瑞典科学家 HÄRKEGÅRD 发表了基于有 限元分析的缺陷萌生疲劳裂纹的研究论文 ${ }^{[168]}$ 。1982 年, 疲劳分析 nCode 公司成立, 其开发的 MSC/FATIGUE 软件系统, 成为开展疲劳有限元分
析的热门工具，使结构疲劳损伤与寿命分析效率和 便捷性显著提高 ${ }^{[169]}$ 。1989 年, MCCLUNG 和 SEHITOGLU ${ }^{[170]}$ 发表了疲劳裂纹闭合模拟的研究成 果, 为人们研究疲劳裂纹扩展驱动力提供了新方向, 至今仍是疲劳有限元分析领域引用次数最高的论 文。20 世纪 90 年代以后, 晶体塑性有限元分析方 法兴起 ${ }^{[171-173]}$, 为人们分析疲劳的微细观损伤提供 了重要手段, 促使材料微观组织敏感的疲劳模拟技 术成为前沿领域 ${ }^{[43,174]}$ 。

近 20 年来,一些新的分析方法的提出满足了疲 
劳多尺度损伤模拟的需求。例如, 分子动力学方法 被用于研究位错与晶界交互作用 ${ }^{[175]}$, 离散位错动力 学方法能够输出滑移和位错密度, 并能得到高度局 部化的塑性应变和永久滑移带信息 ${ }^{[176]}$, 晶体塑性有 限元方法被用于研究晶向和滑移系的影响, 且应变 梯度模型可输出几何必要性位错密度等信息 ${ }^{[177]}$, 扩 展有限元法实现了 $3 \mathrm{D}$ 裂纹扩展模拟, 且使网格划 分更加便利 ${ }^{[178]}$ 。通过发展以断裂机理为基础的疲劳 损伤模拟技术, 并与宏观连续介质模型实现关联, 进而完整呈现疲劳裂纹的萌生过程及寿命, 被认为 是可行的技术路径, 目前是疲劳领域的热门研究方 向。这一方向目前仍存在一些难题, 包括: 分子动 力学模拟与试验存在时间与长度尺度的不匹配性, 人们正尝试采用原位 TEM 与分子动力学的协同方 法来研究变形微观机制 ${ }^{[179]}$; 传统 TEM 的位错观察 仅仅体现了 $2 \mathrm{D}$ 薄膜局部的结果, 导致离散位错动 力学的位错模拟结果难以用传统 TEM 的位错分布 进行验证, 且模拟仅能获得少数循环的结果 ${ }^{[180]}$ 。

\section{4 新起点新路径}

百年疲劳研究的回顾, 从起源与演化过程看理 论与技术贡献, 从文献分析中看热点与趋势, 从㽻 劳的历史变迁中看标志性工作、关键领域的进展与 现状, 以及试验与分析方法的进步, 比较完整地呈 现了疲劳研究的历史、问题和现状。站在新的起点 上, 疲劳研究向何处去成为需要回答的根本问题。 为此, 总结疲劳研究领域的待解难题显得十分必要, 而大数据技术可能为突破现有科学难题提供新的 机遇。

\section{1 疲劳研究领域的待解难题}

\subsection{1 疲劳极限的存在性问题}

一直以来, 人们将疲劳极限作为材料的本质属 性, 认为是材料常数, 用于评价材料优劣, 是结构 抗疲劳设计的重要参数。人们普遍认为它代表的是 裂纹萌生后不发生扩展的临界应力水平。传统认为 裂纹主要萌生于表面, 疲劳极限反映了表面缺陷或 表面裂纹不扩展时的疲劳强度。实际上, 疲劳极限 的尺度特性对其存在性构成挑战, 疲劳极限不仅仅 局限于材料层面, 结构或部件尺度上的疲劳极限对 断裂控制至关重要。此外, 随着超高周疲劳研究的 不断深入, 疲劳破坏常起源于内部缺陷(如夹杂物、 气孔)或不连续组织处, 致使 $S-N$ 曲线呈现多阶段特 征 ${ }^{[181]}$ 。若从材料内部缺陷的角度分析疲劳极限, 则 材料受交变载荷终会断裂于内部缺陷处, 即疲劳极
限是不存在的 ${ }^{[41]}$, 有必要修正传统疲劳设计方法; 若从疲劳损伤过程的角度分析疲劳极限, 含缺陷或 理想材料的疲劳损伤及其裂纹萌生与扩展过程, 必 然存在裂纹是否萌生或扩展的临界应力条件 ${ }^{[182]}$, 即 疲劳极限可能存在。因此, 是否存在真实的疲劳极 限仍是一个需要进一步求证的科学难题 ${ }^{[183]}$ 。

\subsection{2 疲劳损伤的易感基因}

循环塑性的不可逆性是疲劳损伤及裂纹萌生的 源头 ${ }^{[184-185]}$, 人们相继建立了疲劳裂纹萌生的理论 模型 ${ }^{[186]}$, 并从微观组织角度开展多尺度损伤模 拟 ${ }^{[43]}$, 综合运用分子动力学、位错动力学、晶体塑 性和连续介质有限元等多种分析方法, 明确了疲劳 寿命分散性与微观组织的关系 ${ }^{[187]}$, 大量研究工作较 好地揭示了材料的循环变形特征 ${ }^{[188]}$ 以及微观组织 的㽻劳损伤机制 ${ }^{[189-190]}$ 。从材料开发与利用角度, 人们更关心疲劳的易感基因，这对于优化材料冶金 与制造工艺至关重要。目前的困境在于, 复杂微观 组织的疲劳损伤机制难以明确, 单一条件与复杂因 素的疲劳机制可能存在差异，不同尺度上的疲劳损 伤主控机制不同, 导致确定控制疲劳性能的微观组 织困难。因此, 是否存在疲劳的易感基因又是一个 科学难题。近年来, 人们发展了高精度的应变测量 与表征技术, 配以原位观察, 如高精度电子背散射 衍射 ${ }^{[191]}$ 、原位扫描电镜/数字图像相关 ${ }^{[192]}$ 和 $3 \mathrm{D} / 4 \mathrm{D}$ 原位同步辐射技术等 ${ }^{[193-195]}$, 可以得到高分辨率的 应变局部化信息 ${ }^{[196]}$, 为开展诸如板条马氏体类微观 组织的疲劳损伤定位及其演化行为研究提供重要工 具 ${ }^{[197]}$, 能够更精准地建立微观组织与疲劳性能的关 系, 进而为寻找相应尺度上的疲劳易感基因提供技 术方案。

\subsection{3 疲劳裂纹扩展的主控因素}

20 世纪 80 年代以来, 人们对裂尖塑性变形和 尾迹的闭合机制的研究, 形成了裂纹扩展动力与阻 力的理论模型和描述方法 ${ }^{[198]}$ 。然而, 疲劳裂纹是否 扩展本质上是一个多尺度断裂问题, 包括材料原子 尺度的断裂、微观尺度上裂纹与材料特征组织的相 互作用、宏观尺度上裂尖与尾迹力学参量平衡等方 面, 断裂准则的尺度相关性, 导致临界扩展条件的 认识不唯一, 疲劳裂纹尖端控制扩展的主要因素仍 是待解难题。近年来, 人们基于数字图像相关法, 尝试发展可定量的裂尖应变累积准则 ${ }^{[199]}$, 相较现有 基于应力强度扩展准则, 能更好反映其物理机制; 基于同步辐射 $\mathrm{X}$ 射线衍射和计算 $\mathrm{X}$ 射线断层成像技 术, 有助于分析裂尖与尾迹的三维断裂物理机 制 ${ }^{[200]}$; 基于衍射祄度成像技术，分析晶粒尺度上的 
三维损伤与断裂机理 ${ }^{[201]}$, 有利于评判断裂模式与裂 纹临界扩展条件。这些先进的试验测量和表征手段 的兴起, 为疲劳裂纹扩展的多尺度特性及其主控因 素的探索研究提供了较好的条件, 但完全澄清断裂 本质的科研之路还很长。

\section{1 .4 蠕变-疲劳交互效应的多尺度认识}

人们对蠕变、疲劳及其交互作用的研究发展了 诸多理论模型和工程防断方法。现有研究认为, 对 某种材料, 蠕变与疲劳两种载荷模式的交互作用需 要具有一定条件, 即敏感窗口期 ${ }^{[110]}$ 。目前存在的问 题是, 在交互效应的机理认识方面, 仍不明确裂纹 起裂和扩展路径是否存在叠加效应, 而疲劳裂纹扩 展的穿晶模式和蠕变裂纹扩展的沿晶模式是否存在 交互作用仍不明确; 在交互效应的设计策略方面,

ASME code case N-47 中线性叠加的下包络线是否 足够保守或过于保守, 仍缺乏数据支持与验证。解 决此类问题需要获得对蠕变-疲劳交互效应的多尺 度认识。从材料尺度看, 需要了解不同材料交互效 应差异的机理, 掌握晶粒内部如何交互, 以及不同 应力水平与材料的交互问题; 从试样尺度看, 需要 明确不同简化载荷谱下的损伤与寿命, 研究缺口或 多向应力的叠加, 并考察数据之间的关联性; 从部 件尺度看, 由于存在广泛的局部不连续应力问题, 需要发展高效的计算方法, 考虑载荷谱的科学简化, 进而获得结构破坏的临界准则。

\subsection{5 疲劳设计安全系数的物理本质}

安全系数是工程结构设计中材料破坏极限与设 计许用值的比值, 安全系数越大代表着设计安全裕 度越大, 保守性越高。人们在确定安全系数的过程 中, 需要综合考虑材料类型、失效模式、应力分析 精度、制造工艺和经济性等因素。随着计算精度的 提高和制造工艺条件的改善 ${ }^{[202]}$, 安全系数也会发生 变化。以压力容器为例, 美国 ASME 设计标准的安 全系数已由最初的 5 降低到 3.5; 中国 GB150 常规 设计的安全系数从 3 降低到 2.7 , 分析设计也由 2.7 降低到 2.4; 欧盟 EN12335 的安全系数较低, 维持 在 2.4 。目前的安全系数确定具有较强的经验性, 人 们仍然不清楚安全系数数值代表的真正含义, 也没 有关于其物理本质的研究。突破这一难题, 需要揭 示材料失效机制的物理本质, 建立相应的断裂准则, 并纳入试样和部件等多个尺度的断裂风险, 如发展 基于损伤模式的压力容器设计技术 ${ }^{[203]}$ 。对疲劳设计 的安全系数而言, 也受到服役寿命区间的影响, 不 同的设计寿命可能具有不同的失效机制, 例如超高 周疲劳中的多阶段 $S-N$ 曲线关系, 会使安全系数表
现为与服役寿命或损伤机制的相关性 ${ }^{[204-205]}$ 。大数 据科学为解决该难题提供了可能的途径。

\section{2 大数据时代的疲劳强度学}

疲劳强度学涉及材料力学行为、制造工艺、结 构几何参数和失效判据等基本内容。现有的研究思 路主要包括从理论到现象的推演法、从试验现象到 理论的归纳法, 以及基于有限元的模拟仿真方法。 然而, 推演法中的理论推导过程存在多重假设和理 想化等问题, 无法对实际试验现象进行准确描述; 归纳法侧重于模拟条件下的现象分析, 导致理论的 普适性不足; 有限元模拟获取变化规律受限于模型 的可靠性。这些方法通常以物理机制和规律为基础, 并依赖于理想和简化模型, 研究范式上注重因果逻 辑。当涉及到具体应用时, 疲劳机理的尺寸相关性, 材料、制造工艺和服役条件等多变量因素 ${ }^{[206]}$, 以及 对安全系数的过度依赖, 都为结构的安全带来不确 定性。

近年来, 以大数据和机器学习为代表的数据科 学成为新时代科学与工程领域的热门工具 ${ }^{[207]}$, 强调 因素之间的关联性，成为发展数据驱动研究范式和 数据融合强度的基础。大数据时代的结构强度学范 式, 使传统动力与能源革命带来的安全与强度知识 基础, 与信息与数据革命带来的数据利用需求得到 有效融合，不仅有利于解决复杂在役结构的故障诊 断和寿命预测难题，也有利于满足高端工业领域机 械结构的全寿命和高可靠安全保障需求。数据融合 的疲劳强度学已经成为前沿研究领域与学科方向。 大数据时代的疲劳强度学研究思路包括, 健康状态 感知、损伤机理预测和寿命智能管理三个步骤, 最 终实现具有数据支撑的结构疲劳寿命预测及装备智

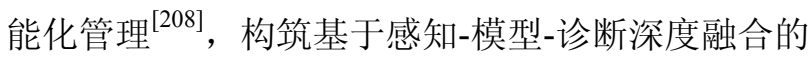
设备服役安全科学基础, 进而提供面向复杂系统服 役安全的一体化方案。

\section{5 结语}

（1）交变往复受载是蒸汽机时代以来机械装备 的固有基因和基本损伤范式, 是动力/能量转换模式 机械化引入的自然结果。随着机器装备的高速、重 载、轻量化等极端化发展, 结构强度安全几余降低, 引发零部件失效事故增多和破坏概率增大, 结构的 疲劳损伤断裂依然是亟待解决的难题。从这一角度 看, 百年疲劳的研究历史是一部科学家、工程师破 解结构疲劳损伤断裂这一 “顽疾” 的奋斗史。

(2) 工程中新的失效模式、新的破坏现象、新 
的研究手段, 一直是推动疲劳强度研究领域新方向 诞生和学科前进的源动力。长期以来, 结构疲劳强 度领域的进展支撑了诸多高端装备和工艺的实现, 然而所建立的上百个寿命预测模型依然局限于试验 数据的拟合或唯象关联, 以及数据小样本和微观分 析的局部性, 仍未能突破疲劳的统计特征和分散性, 难免 “以偏概全”。大量工作局限于材料损伤机理和 试验数据的简单重复, 在物理机制研究和寿命预测 领域引领性和突破性的工作较少体现。

(3) 随着物联网和工业互联网技术的普及, 可 以预见, 未来疲劳领域的工作将不仅仅是传统知识 的工程应用和普及, 数据科学和大数据技术必将在 疲劳寿命精准预测、疲劳损伤机制的微纳观尺度诠 释和结构寿命可靠性实施预警方面具有更大的突破 空间。

\section{参 考 文 献}

[1] ALBERT W A J. Uber Treibseile am Harz Archiv fur Mineralogie, Georgnosie[J]. Bergbau und Huttenkunde, 1837, 10: 215-234.

ALBERT W A J. About driving ropes on the Harz. Archives for mineralogy, georgnosie[J]. Mining and Huttenkunde, 1837, 10: 215-234.

[2] PONCELET $\mathrm{J} \mathrm{V}$. Introduction à la mécanique industrielle, physique ou' expérimentale[M]. Deuxieme ed. Paris: Imprinmerie de Gauthier-Villars, 1839.

PONCELET J V. Introduction to industrial, physical or experimental mechanics[M]. 2nd ed. Paris : Gauthier-Villars Imprinmerie, 1839.

[3] RANKINE W J M. On the causes of the unexpected breakage of the journals of railway axles; and on the means of preventing such accidents by observing the law of continuity in their construction[C]/Minutes of the Proceedings of the Institution of Civil Engineers, Thomas Telford-ICE Virtual Library, 1843: 105-107.

[4] HODGKINSON E A. Report of the commissioners appointed to inquire into the application of iron to railway structures[M]. London: William Clowes and Sons, 1849.

[5] BRAITHWAITE F. On the fatigue and consequent fracture of metals [C]// Minutes of the Proceedings of the Institution of Civil Engineers. Thomas Telford-ICE Virtual Library, 1854, 13(1854): 463-467.

[6] GOUGH H J. The fatigue of metals[M]. London: Scott, Greenwood, 1924.

[7] 高镇同. 疲劳性能测试[M]. 北京: 国防工业出版社,
1980.

GAO Zhentong. Fatigue properties test [M]. Beijing: National Defense Industry Press, 1980.

[8] FAIRBAIRN W. Experiments to determine the effect of impact, vibratory action, and long-continued changes of load on wrought-iron girders[J]. Philosophical Transactions of the Royal Society of London, 1864(154): 311-325.

[9] 颜鸣㚖. 金属疲劳裂纹扩展规律及其微观机制(上) $[\mathrm{J}]$. 航空材料, 1978(5): 1-10.

YAN Minggao. Fatigue crack growth law and its micromechanism of metals (1)[J]. Journal of Aeronautical Materials, 1978(5): 1-10.

[10] ZHANG W. Technical problem identification for the failures of the liberty ships[J]. Challenges, 2016，7(2): 20.

[11] STEPHENS R I, FATEMI A, STEPHENS R R, et al. Metal fatigue in engineering[M]. New York: John Wiley \& Sons, 2000.

[12] IRWIN G. Discussion of the dynamic stress distribution surrounding a running crack-A photoelastic analysis[J]. Proceedings of the Society for Experimental Stress Analysis, 1958, 16(1): 93-96.

[13] PARIS P, ERDOGAN F. A critical analysis of crack propagation laws[J]. Journal of Basic Engineering, 1963, 85: $528-534$.

[14] ELBER W. The significance of fatigue crack closure[M]//Damage tolerance in aircraft structures. West Conshohocken, Pennsylvania: ASTM International, 1971.

[15] 俞茂宏, 彭一江. 强度理论百年总结[J]. 力学进展, 2004, 34(4): 529-560.

YU Maohong, PENG Yijiang. Advances in strength theorys for materials under complex stress-strain in the 20th century[J]. Advances in Mechanics, 2004, 34(4): 529-560.

[16] 张小丽, 陈雪峰, 李兵, 等. 机械重大装备寿命预测综 述 $[\mathrm{J}]$. 机械工程学报, 2011，47(11): 100-116.

ZHANG Xiaoli, CHEN Xuefeng, LI Bing, et al. Review of life prediction for mechanical major equipments[J]. Journal of Mechanical Engineering, 2011, 47(11): 100-116.

[17] MILLER K J, 柯伟，韩玉梅，等. 金属疲劳——过去、 现在和未来 $(一)[\mathrm{J}]$. 机械强度, 1993(1): 77-80, 61 . MILLER K J, KE Wei, HAN Yumei, et al. Metal fatigue: past, present and future (1)[J]. Journal of Mechanical 
Strength, 1993(1): 77-80, 61.

[18] WÖHLER A. Wöhler's experiments on the strength of metals[J]. Engineering, 1867, 4: 160-161.

[19] WÖHLER A. Über die festigkeitsversuche mit eisen und stahl[J]. Zeitschrift für Bauwesen, 1870, 20: 73-106.

WÖHLER A. About the strength tests with iron and steel [J]. Construction Journal, 1870, 20: 73-106.

[20] BAUSCHINGER J. Ueber die Veranderung der elasticitatsgrenge und der festigkeit des eisens und stahls durch strecken und quetschn, durch erwarmen und abkuhlen und durch oftmal wiederholte beanspruchung[J]. Mitteilungen aus dem Mechanisch-Technischen Laboratorium der $\mathrm{K}$, Technischen Hochschule in Munchen, 1886, 13: 1-115.

BAUSCHINGER J. About the change in elasticity and the strength of iron and steel through stretching and squeezing, through heating and cooling and through often repeated stress $[\mathrm{J}]$. Reports from the mechanical-technical laboratory of the K, Technical University in Munich, 1886, 13: 1-115.

[21] BASQUIN O. The exponential law of endurance tests [C]//Proc. Am. Soc. Test. Mater., 1910, 10: 625-630.

[22] PALMGREN A. Die lebensdauer von kugellagern[J]. Zeitschrift des Vereinesdeutscher Ingenierure, 1924, 68(14): 339-341.

PALMGREN A. The life of ball bearings[J]. Journal of the Association of German Engineers, 1924, 68(14): 339-341.

[23] MINER M A. Cumulative damage in fatigue[J]. Journal of Applied Mechanics, 1945, 12: 159-164.

[24] MANSON S S. Behavior of materials under conditions of thermal stress[R]. National Advisory Committee for Aeronautics, 1953(2933).

[25] COFFIN JR L F. A study of the effects of cyclic thermal stresses on a ductile metal[J]. Transactions of the American Society of Mechanical Engineers, 1954, 76: 931-950.

[26] FORSYTH P J E. The physical basis of metal fatigue[M]. London: Blackie and Son Ltd., 1969.

[27] RYDER D A. Some quantitative information obtained from the examination of fatigue fracture surfaces[R]. Technical Note No: MET 288. Farnborough, UK: Royal Aircraft Establishment, 1958.

[28] NEUBER H. Theory of notch stresses: Principles for exact calculation of strength with reference to structural form and material[R]. USAEC Office of Technical Information, 1961(4547).

[29] MATSUISHI M, ENDO T. Fatigue of metals subjected to varying stress[J]. Japan Society of Mechanical Engineers, 1968, 68(2): 37-40.

[30] BROWN M W, MILLER K J. A theory for fatigue failure under multiaxial stress-strain conditions[J]. Proceedings of the Institution of Mechanical Engineers, 1973, 187(1): 745-755.

[31] MILLER K. The short crack problem[J]. Fatigue \& Fracture of Engineering Materials \& Structures, 1982, 5(3): 223-232.

[32] LANKFORD J. The growth of small fatigue cracks in 7075-T6 aluminum[J]. Fatigue \& Fracture of Engineering Materials \& Structures, 1982, 5(3): 233-248.

[33] SURESH S, RITCHIE R. Propagation of short fatigue cracks[J]. International Metals Reviews, 1984, 29(1): 445-475.

[34] RITCHIE R, LANKFORD J. Small fatigue cracks: a statement of the problem and potential solutions $[\mathrm{J}]$. Materials Science and Engineering, 1986, 84: 11-16.

[35] RITCHIE R. Near-threshold fatigue-crack propagation in steels[J]. International Metals Reviews, 1979, 24(1): 205-230.

[36] SURESH S , ZAMISKI G F , RITCHIE $\mathrm{R} O$. Oxide-induced crack closure : An explanation for near-threshold corrosion fatigue crack growth behavior[J]. Metallurgical and Materials Transactions A, 1981, 12(8): 1435-1443.

[37] SURESH S, RITCHIE R. A geometric model for fatigue crack closure induced by fracture surface roughness[J]. Metallurgical Transactions A, 1982, 13(9): 1627-1631.

[38] 高镇同, 傅惠民. 疲劳强度的频率分布和疲劳强度特征 函数 $[\mathrm{J}]$. 机械强度, 1985(2): 27-34.

GAO Zhentong, FU Huimin. Frequency distribution and characteristic function of fatigue strength[J]. Journal of Mechanical Strength, 1985(2): 27-34.

[39] 阎楚良, 王公权. 峰值计数法疲劳载荷谱的编制与统计 处理程序[J]. 农业机械学报, 1984(1): 51-57.

YAN Chuliang, WANG Gongquan. Compilation and statistical processing program of fatigue load spectrum by peak count method[J]. Transactions of the Chinese Society of Agricultural Machinery, 1984(1): 51-57.

[40] 欧进萍, 叶骏. 结构风振的概率疲劳累积损伤 [J]. 振动 工程学报, 1993, 6(2): 164-169. 
OU Jinping, YE Jun. Probabilistic fatigue cumulative damage of wind-induced structures[J]. Journal of Vibration Engineering, 1993，6(2): 164-169.

[41] BATHIAS C. There is no infinite fatigue life in metallic materials[J]. Fatigue \& Fracture of Engineering Materials \& Structures, 1999, 22(7): 559-565.

[42] MURAKAM Y, NOMOTO T, UEDA T. Factors influencing the mechanism of superlong fatigue failure in steels[J]. Fatigue \& Fracture of Engineering Materials \& Structures, 1999, 22(7): 581-590.

[43] MCDOWELL D L, DUNNE F P E. Microstructuresensitive computational modeling of fatigue crack formation[J]. International Journal of Fatigue, 2010, 32(9): 1521-1542.

[44] GOODMAN J. Mechanics applied to engineering[M]. London: Longmans, Green, 1919.

[45] History of SATEC, A rich history of materials testing equipment[EB/OL]. [2005-02-01]. Grove City, PA : Instron, 2005. https://www.instron.us/-/media/literaturelibrary/corporate/2005/02/history-of-satec.pdf.

[46] SMITH R, WATSON P, TOPPER T. A stress-strain parameter for the fatigue of metals[J]. Journal of Materials, 1970, 5(4): 767-778.

[47] FATEMI A, SOCIE D F. A critical plane approach to multiaxial fatigue damage including out-of-phase loading[J]. Fatigue \& Fracture of Engineering Materials \& Structures, 1988, 11(3): 149-165.

[48] 颜鸣臬. 金属疲劳裂纹扩展规律及其微观机制(下) $[\mathrm{J}]$. 航空材料, 1979(1): 29-45, 28.

YAN Minggao. Fatigue crack growth law and its micromechanism of metals (3)[J]. Journal of Aeronautical Materials, 1979(1): 29-45, 28.

[49] 颜鸣㚖. 金属疲劳裂纹扩展规律及其微观机制(中) $[\mathrm{J}]$. 航空材料, 1978(6): 38-47.

YAN Minggao. Fatigue crack growth law and its micromechanism of metals (2)[J]. Journal of Aeronautical Materials, 1978(6): 38-47.

[50] 章安庆, 孔庆平, 师昌绪. 一种镍基合金的蠕变和低周 疲劳的交互作用[J]. 金属学报, 1979(4): 518-525, 592. ZHANG Anqing, KONG Qingping, SHI Changxu. Interaction between creep and low cycle fatigue of a nickel base alloy[J]. Acta Metallurgica Sinica, 1979(4): 518-525, 592.

[51] 徐影. 疲劳强度的可靠性设计 [J]. 东北工学院学报, 1979(2): 82-91.
XU Hao. Reliability design of fatigue strength[J]. Journal of Northeast Institute of Technology， 1979(2): 82-91.

[52] 张康达. 压力容器疲劳失效的可靠性估计 [J]. 浙江化 工学院学报, 1979(2): 4-13.

ZHANG Kangda. Reliability estimation of pressure vessel fatigue failure[J]. Journal of Zhejiang University of Chemical Technology, 1979(2): 4-13.

[53] 六二一所 X 光结构分析组, 六二一所喷丸强化组. 喷 丸强化对镍基高温合金及燃气浴轮叶片高温疲劳性能 的影响[J]. 航空材料, 1979(1): 15-19, 47.

X-ray Structural Analysis Group of 621 Institute, Shot Peening Group of 621 Institute. Effect of shot peening on high temperature fatigue properties of nickel base superalloy and gas turbine blade[J]. Journal Aeronautical Materials, 1979(1): 15-19, 47.

[54] BARSAM J M. 合肥通用机械研究所断裂㓞性课题组. 压力容器用钢的疲劳行为 $(一)[\mathrm{J}]$. 化工与通用机械, 1975(10): 48-62.

BARSAM J M. Fracture Toughness Research Group, Hefei General Machinery Research Institute. Fatigue behavior of pressure vessel steel (1)[J]. Chemical Industry and General Machinery, 1975(10): 48-62.

[55] 中国科学院北京力学研究所十二室疲劳组. 金属疲劳 中的累积损伤理论[J]. 力学情报, 1976(1): 53-61.

Fatigue Group, Department 12, Beijing Institute of Mechanics, Chinese Academy of Sciences. Cumulative damage theory in metal fatigue[J]. Journal of Mechanical Information, 1976(1): 53-61.

[56] 郑修麟. 循环局部应力-应变与疲劳裂纹起始寿命 [J]. 固体力学学报, 1984(2): 175-184.

ZHENG Xiulin. Cyclic local stress-strain and fatigue crack initiation life[J]. Acta Mechnica Solida Sinica, 1984(2): 175-184.

[57] 阎楚良. 双参数疲劳载荷谱的编制 $[\mathrm{J}]$. 农业机械学报, 1986(2): 94-102.

YAN Chuliang. Investigation of compilation of fatigue load spectrum by two- parametric counting method[J]. Transactions of The Chinese Society of Agricultural Machinery, 1986(2): 94-102.

[58] 谢里阳, 林文强. 线性累积损伤的概率准则 [J]. 机械强 度, 1993, 15(3): 41-44.

XIE LiYang, LIN Wenqiang. Probabilistic linear cumulative fatigue damage rule[J]. Journal of Mechanical Strength, 1993, 15(3): 41-44.

[59] 李金鬼, 李海涛, 姚枚, 等. 喷丸产生的残余拉应力场 
及材料的内部疲劳极限 [J]. 航空学报, 1990, 11(7): 369-375.

LI Jinkui, LI Haitao, YAO Mei, et al. Investigation on the tensile residual stress due to shot peening and the internal fatigue limit of metals[J]. Acta Aeronautica et Astronautica Sinica, 1990, 11(7): 369-375.

[60] 王仁智, 汝继来, 李向斌, 等. 疲劳裂纹萌生的微细观 过程与内部疲劳极限理论[J]. 金属热处理学报, 1995, 16(4): 26-34.

WANG Renzhi, RU Jilai, LI Xiangbin, et al. A micro-meso-process theory of fatigue crack initiation and theory of internal fatigue limit[J]. Transactions of Metal Heat Treatment, 1995, 16(4): 26-34.

[61] 陈旭, 高庆, 孙训方, 等. 非比例载荷下多轴低周疲劳 研究最新进展[J]. 力学进展, 1997, 27(3): 26-38.

CHEN Xu, GAO Qing, SUN Xunfang, et al. Recent advances of multiaxial low-cycle fatigue under nonproportional loading[J]. Advances in Mechanics, 1997, 27(3): 26-38.

[62] 姚卫星. 金属材料疲劳行为的应力场强法描述 [J]. 固 体力学学报, 1997, 18(1): 38-48.

YAO Weixing. The description for fatigue behaviours of metals by stress field intensity approach[J]. Acta Mechnica Solida Sinica, 1997, 18(1): 38-48.

[63] 尚德广, 王大康, 李明, 等. 随机疲劳寿命预测的局部 应力应变场强法[J]. 机械工程学报, 2002, 38(1): 67-70. SHANG Deguang, WANG Dakang, LI Ming, et al. Method of local stress-strain fieid intensity for random fatigue life prediction[J]. Journal of Mechanical Engineering, 2002, 38(1): 67-70.

[64] 尚德广, 王德俊, 姚卫星. 多轴非线性连续疲劳损伤累 积模型的研究 [J]. 固体力学学报, 1999, 20(4): 325-330. SHANG Deguang, WANG Dejun, YAO Weixing. Study on nonlinear continuum multiaxial fatigue cumulative damage model[J]. Acta Mechnica Solida Sinica, 1999, 20(4): $325-330$.

[65] 崔维成, 蔡新刚, 冷建兴. 船舶结构疲劳强度校核研究 现状及我国的进展 $[J]$. 船舶力学，1998，2(4)：63-81. CUI Weicheng, CAI Xingang, LENG Jianxing. A state of the art review for the fatigue strength assessment of ship structures[J]. Journal of Ship Mechanics, 1998, 2(4): 63-81.

[66] 王成国, 孟广伟, 原亮明, 等. 新型高速客车构架的疲 劳寿命数值仿真分析 [J]. 中国铁道科学, 2001, 22(3): 94-98.
WANG Chengguo , MENG Guangwei , YUAN Liangming, et al. The analysis of fatigue life of a new type of high-speed passenger car's frame[J]. China Railway Science, 2001, 22(3): 94-98.

[67] 金学松, 沈志云. 轮轨滚动接触疲劳问题研究的最新进 展 $[\mathrm{J}]$. 铁道学报, 2001, 23(2): 92-108.

JIN Xuesong, SHEN Zhiyun. Rolling contact fatigue of wheel/rail and its advanced research progress[J]. Journal of the China Railway Society，2001，23(2): 92-108.

[68] 葛修润, 蒋宇, 卢允德, 等. 周期荷载作用下岩石疲劳 变形特性试验研究 [J]. 岩石力学与工程学报, 2003, 22(10): 1581-1585.

GE Xiurun, JIANG Yu, LU Yunde, et al. Testing study on study on fatigue deformation law of rock under cyclic loading $[\mathrm{J}]$. Chinese Journal of Rock Mechanics and Engineering, 2003, 22(10): 1581-1585.

[69] 蒋宇, 葛修润, 任建喜. 岩石疲劳破坏过程中的变形规 律及声发射特性 [J]. 岩石力学与工程学报, 2004, 23(11): 1810-1814.

JIANG Yu, GE Xiurun, REN Jianxi. Deformation results and acoustic emission characteristics of rock in process of fatigue failure[J]. Chinese Journal of Rock Mechanics and Engineering, 2004, 23(11): 1810-1814.

[70] 李德源, 叶枝全, 陈严, 等. 风力机叶片载荷谱及疲劳 寿命分析[J]. 工程力学，2004，21(6): 118-123.

LI Deyuan, YE Zhiquan, CHEN Yan, et al. Load spectrum and fatigue life analysis of the blade of horizontal axis wind turbine[J]. Engineering Mechanics, 2004, 21(6): 118-123.

[71] 毛喆, 初秀民, 严新平, 等. 汽车驾驶员驾驶疲劳监测 技术研究进展 $[\mathrm{J}]$. 中国安全科学学报, 2005, 15(3): 108-112.

MAO Zhe, CHU Xiumin, YAN Xinping, et al. Advances of fatigue detecting technology for drivers $[\mathrm{J}]$. China Safety Science Journal， 2005， 15(3): 108-112.

[72] 冯国庆. 船舶结构疲劳强度评估方法研究[D]. 哈尔滨: 哈尔滨工程大学, 2006.

FENG Guoqing. Research on fatigue strength evaluation method of ship structure[D]. Harbin: Harbin Engineering University, 2006.

[73] 赵永翔. 应变疲劳可靠性分析的现状及展望 [J]. 机械 工程学报, 2001，37(11): 1-6.

ZHAO Yongxiang. On the strain based fatigue reliability analysis[J]. Journal of Mechanical Engineering, 2001, 37(11): 1-6. 
[74] 王清远. 超声加速疲劳实验研究 [J]. 四川大学学报, 2002, 34(3): 6-11.

WANG Qingyuan. Accelerated fatigue testing by ultrasonic loading $[\mathrm{J}]$. Journal of Sichuan University,

2002, 34(3): 6-11.

[75] 薜红前, 陶华. $20 \mathrm{kHz}$ 频率下高强度钢超高周疲劳研究 [J]. 机械工程材料, 2005，29(5): 12-15.

XUE Hongqian, TAO Hua. Super high cycle fatigue of high strength steels at a frequency of $20 \mathrm{kHz}[\mathrm{J}]$. Materials for Mechanical Engineering, 2005，29(5): 12-15.

[76] 刘龙隆, 轩福贞, 朱明亮. $25 \mathrm{Cr} 2 \mathrm{Ni} 2 \mathrm{MoV}$ 钢焊接接头的 超高周疲劳特性[J]. 机械工程学报, 2014, 50(4): 25-31. LIU Longlong, XUAN Fuzhen, ZHU Mingliang. Very-high cycle fatigue behavior of $25 \mathrm{Cr} 2 \mathrm{Ni} 2 \mathrm{MoV}$ steel welded joint[J]. Journal of Mechanical Engineering, 2014, 50(4): 25-31.

[77] 王弘, 高庆. 40Cr 钢超高周疲劳性能及疲劳断口分析 [J]. 中国铁道科学, 2003, 24(6): 94-99.

WANG Hong, GAO Qing. Study on the gigacycle fatigue behaviors and fracture surface of $40 \mathrm{Cr}$ steel[J]. China Railway Science, 2003, 24(6): 94-99.

[78] 张继明, 杨振国, 李守新, 等. 汽车用高强度弹簧钢 $54 \mathrm{SiCrV} 6$ 和 $54 \mathrm{SiCr} 6$ 的超高周疲劳行为 $[\mathrm{J}]$. 金属学报, 2006, 42(3): 259-264.

ZHANG Jiming, YANG Zhenguo, LI Shouxin, et al. Ultra-high cycle fatigue behavior of automotive high strength spring steels 54SiCrV6 AND 54SiCr6[J]. Acta Metallurgica Sinica, 2006, 42(3): 259-264.

[79] 周承恩, 洪友士. GCr15 钢超高周疲劳行为的实验研究 $[\mathrm{J}]$. 机械强度，2004，26(S1): 157-160.

ZHOU Chengen , HONG Youshi. Experimental investigation on very-high cycle fatigue of $15 \mathrm{Cr}$ steel[J]. Journal of Mechanical Strength, 2004, 26(S1): 157-160.

[80] 赵振业. 高强度合金抗疲劳应用技术研究与发展 [J]. 中国工程科学, 2005, 7(3): 90-94.

ZHAO Zhenye. Investigation and development status of the application technology to improve fatigue behavior of high strength alloys[J]. Engineering Science, 2005, 7(3): 90-94.

[81] 吴学仁, 刘建中. 基于小裂纹理论的航空材料疲劳全寿 命预测[J]. 航空学报, 2006, 27(2): 219-226.

WU Xueren, LIU Jianzhong. Total fatigue life prediction for aeronautical materials by using small-crack theory[J]. Acta Aeronautica et Astronzutica Sinica, 2006, 27(2): 219-226.
[82] 谢里阳, 任俊刚, 吴宁祥, 等. 复杂结构部件概率疲劳 寿命预测方法与模型 [J]. 航空学报, 2015, 36(8): 2688-2695.

XIE Liyang, REN Jungang, WU Ningxiang, et al. Probabilistic fatigue life prediction method and modeling for complex structural parts[J]. Acta Aeronautica et Astronautica Sinica, 2015, 36(8): 2688-2695.

[83] 邓扬, 李爱群, 刘扬, 等. 钢桥疲劳荷载效应监测数据 概率建模与疲劳可靠性分析方法 [J]. 土木工程学报, 2014, 47(7): 79-87.

DENG Yang, LI Aiqun, LIU Yang, et al. Probabilistic modeling of fatigue loading effects and fatigue reliability evaluation for steel bridges based on monitored data[J]. China Civil Engineering Journal， 2014， 47(7): 79-87.

[84] 高阳, 白广忱, 张瑛莉. 浴轮盘低循环疲劳寿命的概率 分析 [J]. 航空动力学报, 2009, 24(4): 804-809.

GAO Yang, BAI Guangchen, ZHANG Yingli. Probability analysis of low cycle fatigue life of turbine $\operatorname{disk}[\mathrm{J}]$. Journal of Aerospace Power, 2009， 24(4): 804-809.

[85] 赵永翔, 杨冰, 何朝明, 等. LZ50 钢概率疲劳 S-N 曲 线外推新方法[J]. 铁道学报, 2004, 26(3): 20-25.

ZHAO Yongxiang, YANG Bing, HE Chaoming, et al. Reasonable extrapolation of the commonly probabilistic fatigue S-N curves into the long life regime for LZ50 axle steel[J]. Journal of the China Railway Society, 2004, 26(3): 20-25.

[86] PEARSON S. Initiation of fatigue cracks in commercial aluminium alloys and the subsequent propagation of very short cracks[J]. Engineering Fracture Mechanics, 1975, $7(2): \quad 235-247$.

[87] KITAGAWA H. Applicability of fracture mechanics to very small cracks or the cracks in the early stage $[\mathrm{C}] /$ Proc. of 2nd ICM, Cleveland, 1976: 627-631.

[88] EL HADDAD M H, TOPPER T, SMITH K. Prediction of non propagating cracks[J]. Engineering Fracture Mechanics, 1979, 11(3): 573-584.

[89] EL HADDAD M H, SMITH K N, TOPPER T H. Fatigue crack propagation of short cracks $[\mathrm{J}]$. Journal of Engineering Materials and Technology, 1979, 101(1): 42-46.

[90] LIAW P, LEAX T, WILLIAMS R, et al. Influence of oxide-induced crack closure on near-threshold fatigue crack growth behavior[J]. Acta Metallurgica, 1982, 30(12): 2071-2078.

[91] NAVARRO A, RIOS E D. A model for short fatigue 
crack propagation with an interpretation of the short-long crack transition[J]. Fatigue \& Fracture of Engineering Materials \& Structures, 1987, 10(2): 169-186.

[92] NAVARRO A, DE LOS RIOS E. Fatigue crack growth modelling by successive blocking of dislocations[J]. Proceedings of the Royal Society of London. Series A: Mathematical and Physical Sciences, 1992, 437(1900): 375-390.

[93] NEWMAN Jr J, PHILLIPS E P, SWAIN M. Fatigue-life prediction methodology using small-crack theory[J]. International Journal of Fatigue, 1999, 21(2): 109-119.

[94] NEWMAN J, WU X, SWAIN M, et al. Small-crack growth and fatigue life predictions for high-strength aluminium alloys. Part II: crack closure and fatigue analyses[J]. Fatigue \& Fracture of Engineering Materials \& Structures, 2000, 23(1): 59-72.

[95] ZHU M L, XUAN F Z, TU S T. Effect of load ratio on fatigue crack growth in the near-threshold regime: A literature review, and a combined crack closure and driving force approach[J]. Engineering Fracture Mechanics, 2015, 141: 57-77.

[96] ZHU M L, XUAN F Z, TU S T. Interpreting load ratio dependence of near-threshold fatigue crack growth by a new crack closure model[J]. International Journal of Pressure Vessels and Piping, 2013, 110: 9-13.

[97] MANSON S, HALFORD G, HIRSCHBERG M. Creep fatigue analysis by strain-range partitioning[R]. NASA TM X-67838, Lewis Research Center, 1971.

[98] LEMAITRE J, CHABOCHE J. A non-linear model of creep-fatigue damage cumulation and interaction(for hot metallic structures)[C]//Mechanics of Visco-elastic Media and Bodies. Gothenburg, Sweden, Springer, 1975: 291-301.

[99] COFFIN L. Concept of frequency separation in life prediction for time-dependent fatigue[R]. General Electric Co., Schenectady, NY, USA, 1976.

[100] HALES R. A quantitative metallographic assessment of structural degradation of type 316 stainless steel during creep - fatigue[J]. Fatigue \& Fracture of Engineering Materials \& Structures, 1980, 3(4): 339-356.

[101] TAKAHASHI Y, DOGAN B, GANDY D. Systematic evaluation of creep-fatigue life prediction methods for various alloys[J]. Journal of Pressure Vessel Technology, 2013, 135(6): 1461-1470.
[102] LANDES J, BEGLEY J. A fracture mechanics approach to creep crack growth[C]// Mechanics of crack growth. West Conshohocken, Pennsylvania : ASTM International, 1976.

[103] BASSANI J, MCCLINTOCK F. Creep relaxation of stress around a crack tip[J]. International Journal of Solids and Structures, 1981, 17(5): 479-492.

[104] SAXENA A. Creep crack growth under non-steady-state conditions $[\mathrm{C}] / /$ Fracture Mechanics : Seventeenth Volume. West Conshohocken, Pennsylvania: ASTM International, 1986.

[105] YOON K B, SAXENA A, MCDOWELL D L. Influence of crack-tip cyclic plasticity on creep-fatigue crack growth[C]//Fracture Mechanics : Twenty-Second Symposium. 1990, 1: 367-392.

[106] XUAN F Z, TU S T, WANG Z. C* estimation for cracks in mismatched welds and finite element validation[J]. International Journal of Fracture, 2004, 126(3): 267-280.

[107] XUAN F Z, SI J, TU S T. Evaluation of C* integral for interacting cracks in plates under tension[J]. Engineering Fracture Mechanics, 2009, 76(14): 2192-2201.

[108] DOWLING A R, TOWNLEY C H A. The effect of defects on structural failure: A two-criteria approach[J]. International Journal of Pressure Vessels and Piping, 1975, 3(2): 77-107.

[109] 涂善东, 轩福贞, 王卫泽. 高温蠕变与断裂评价的若 干关键问题[J]. 金属学报, 2009, 45(7): 781-787.

TU Shantung, XUAN Fuzhen, WANG Weize. Some critaical issues in creep and fracture assessment at high temperature[J]. Acta Metallurgica Sinica, 2009, 45(7): 781-787.

[110] HALES R. A method of creep damage summation based on accumulated strain for the assessment of creep-fatigue endurance[J]. Fatigue \& Fracture of Engineering Materials \& Structures, 1983, 6(2): 121-135.

[111] HALES R. The role of cavity growth mechanisms in determining creep-rupture under multiaxial stresses[J]. Fatigue \& Fracture of Engineering Materials \& Structures, 1994, 17(5): 579-591.

[112] GONG J G, XUAN F Z. Notch behavior of components under the stress-controlled creep-fatigue condition: Weakening or strengthening?[J]. Journal of Pressure Vessel Technology, 2017, 139(1): 011407.

[113] SHIPILOV S A. 应力腐蚀破裂和腐蚀疲劳研究: 历史 回顾与趋势 $[C] / / 2002$ 中国国际腐蚀控制大会, 中国北 
京, 2002.

SHIPILOV S A. A research on stress corrosion cracking and corrosion fatigue: historical review and trends[C]// 2002 China International Conference on Corrosion Control, Beijing, China, 2002.

[114] HENDRICKS W R. The Aloha airlines accident - a new era for aging aircraft[M]//Structural integrity of aging airplanes. Springer, Berlin, Heidelberg, 1991: 153-165.

[115] SHIPILOV S A. Stress corrosion cracking, corrosion fatigue and hydrogen embrittlement research[C]// Environmentally Induced Cracking of Metals, Canadian Institute of Mining, Metallurgy and Petroleum, Montreal, Quebec, Canada, 2000.

[116] LECKIE H P. Effects of environments on stress induced failure of high strength maraging steels[C]// Proceedings of Conference on Fundamental Aspects of Stress Corrosion Cracking, National Association of Corrosion Engineers, 1969: 411.

[117] WEI R P, LANDES J D. The effect of D2O on fatigue-crack propagation in a high-strength aluminum alloy[J]. International Journal of Fracture Mechanics, 1969, 5(1): 69-71.

[118] WEI R, LANDES J. Correlation between sustained-load and fatigue crack growth in high-strength steels[J]. Materials Research and Standards, 1972, 12: 45-56.

[119] WEI R P. On understanding environment-enhanced fatigue crack growth-A fundamental approach[C]// Fatigue Mechanisms. West Conshohocken , Pennsylvania: ASTM International, 1979.

[120] AUSTEN I M , WALKER E F. Quantitative understanding of the effects of mechanical and environmental variables on corrosion fatigue crack growth behaviour[C]//The Influence of Environment on Fatigue. London: Institution of Mechanical Engineers, 1977.

[121] 韩恩厚. 核电站关键材料在微纳米尺度上的环境损伤 行为研究-进展与趋势 $[J]$. 金属学报, 2011, 47(7): 769-776.

HAN Enhou. Research trends on micro and nano-scale materials degraadation in nuclear power plant[J]. Acta Metallurgica Sinica, 2011, 47(7): 769-776.

[122] NAITO T, UEDA H, KIKUCHI M. Fatigue behavior of carburized steel with internal oxides and nonmartensitic microstructure near the surface[J]. Metallurgical Transactions A, 1984, 15(7): 1431-1436.
[123] PYTTEL B, SCHWERDT D, BERGER C. Very high cycle fatigue-Is there a fatigue limit?[J]. International Journal of Fatigue, 2011， 33(1): 49-58.

[124] CHANDRAN K S R. Duality of fatigue failures of materials caused by Poisson defect statistics of competing failure modes[J]. Nature Materials, 2005, 4(4): 303-308.

[125] RAVI CHANDRAN K S, JHA S K. Duality of the S-N fatigue curve caused by competing failure modes in a titanium alloy and the role of Poisson defect statistics[J]. Acta Materialia, 2005, 53(7): 1867-1881.

[126] 李守新. 高强度钢超高周疲劳性能: 非金属夹杂物的 影响[M]. 北京：治金工业出版社, 2010.

LI Shouxin. Ultra-high cycle fatigue property of high strength steel: Influence of non-metallic inclusions[M]. Beijing: Metallurgical Industry Press, 2010.

[127] 洪友士, 孙成奇, 刘小龙. 合金材料超高周疲劳的机 理与模型综述 $[\mathrm{J}]$. 力学进展, 2018，48：201801.

HONG Youshi, SUN Chengqi, LIU Xiaolong. A review on mechanisms and models for very-high-cycle fatigue of metallic materials[J]. Advances in Mechanics, 2018, 48: 201801.

[128] MARINES I, BIN X, BATHIAS C. An understanding of very high cycle fatigue of metals $[\mathrm{J}]$. International Journal of Fatigue, 2003， 25(9-11): 1101-1107.

[129] LI S. Effects of inclusions on very high cycle fatigue properties of high strength steels[J]. International Materials Reviews, 2012, 57(2): 92-114.

[130] WANG Q Y, BATHIAS C, KAWAGOISHI N, et al. Effect of inclusion on subsurface crack initiation and gigacycle fatigue strength[J]. International Journal of Fatigue, 2002, 24(12): 1269-1274.

[131] SHIOZAWA K, MORII Y, NISHINO S, et al. Subsurface crack initiation and propagation mechanism in high-strength steel in a very high cycle fatigue regime $[\mathrm{J}]$. International Journal of Fatigue, 2006, 28(11): 1521-1532.

[132] SAKAI T, HARADA H, OGUMA N. Crack initiation mechanism of bearing steel in very high cycle fatigue[J/CD]//Proceedings of ECF-16, CD-ROM, 2006.

[133] SAKAI T, SATO Y, NAGANO Y, et al. Effect of stress ratio on long life fatigue behavior of high carbon chromium bearing steel under axial loading $[\mathrm{J}]$. International Journal of Fatigue, 2006, 28(11): 1547-1554. 
[134] SAKAI T. Review and prospects for current studies on very high cycle fatigue of metallic materials for machine structural use[J]. Journal of Solid Mechanics and Materials Engineering, 2009，3(3): 425-439.

[135] GRAD P, REUSCHER B, BRODYANSKI A, et al. Mechanism of fatigue crack initiation and propagation in the very high cycle fatigue regime of high-strength steels[J]. Scripta Materialia, 2012, 67(10): 838-841.

[136] CHAI G, FORSMAN T, GUSTAVSSON F, et al. Formation of fine grained area in martensitic steel during very high cycle fatigue[J]. Fatigue \& Fracture of Engineering Materials \& Structures, 2015, 38(11): $1315-1323$

[137] CHAI G, ZHOU N, CIUREA S, et al. Local plasticity exhaustion in a very high cycle fatigue regime[J]. Scripta Materialia, 2012, 66(10): 769-772.

[138] HONG Y, LIU X, LEI Z, et al. The formation mechanism of characteristic region at crack initiation for very-high-cycle fatigue of high-strength steels[J]. International Journal of Fatigue， 2016， 89: 108-118.

[139] CHANG Y K, PAN X N, ZHENG L, et al. Microstructure refinement and grain size distribution in crack initiation region of very-high-cycle fatigue regime for high-strength alloys[J]. International Journal of Fatigue, 2020, 134: 12.

[140] ZHU M L, JIN L, XUAN F Z. Fatigue life and mechanistic modeling of interior micro-defect induced cracking in high cycle and very high cycle regimes[J]. Acta Materialia, 2018, 157: 259-275.

[141] ZHU M L, XUAN F Z. Failure mechanisms and fatigue strength assessment of a low strength $\mathrm{Cr}-\mathrm{Ni}-\mathrm{Mo}-\mathrm{V}$ steel welded joint: Coupled frequency and size effects[J]. Mechanics of Materials， 2016， 100: 198-208.

[142] Leading through specialization : RUMUL resonant fatigue testing machines[EB/OL]. [2011-04]. https:// www.rumul.ch/110_cn_firma.php.

[143] BATHIAS C, PARIS P C. Gigacycle fatigue in mechanical practice[M]. New York: Marcel Dekker, 2005.

[144] STANZL-TSCHEGG S. Very high cycle fatigue measuring techniques[J]. International Journal of Fatigue, 2014, 60: 2-17.

[145] EWING J, HUMFREY J. The fracture of metals under repeated alternations of stress[J]. Proceedings of the Royal Society of London. Series A, Mathematical and
Physical Sciences, 1903, 200: 241.

[146] WISCHNITZER S. Introduction to electron microscopy [M]. New York: Elsevier, 2013.

[147] 赵子华, 张峥, 吴素君, 等. 金属疲劳断口定量反推 研究综述 $[J]$. 机械强度, 2008，30(3): 508-514.

ZHAO Zihua, ZHANG Zheng, WU Sujun, et al. Survey of quantitative reverse deduction from fatigue fracture surface of metals[J]. Journal of Mechanical Strength, 2008, 30(3): 508-514.

[148] AKITA K, YOSHIOKA Y, SUZUKI H, et al. X-ray fractography using synchrotron radiation - Residual stress distribution just beneath fatigue fracture surface $[\mathrm{J}]$. Materials Science Research International，2000，6(4): 269-274.

[149] SAVELLI S, BUFFIERE J Y, FOUGERES R. Pore characterization in a model cast aluminum alloy and its quantitative relation to fatigue life studied by synchrotron X-ray microtomography[C]//Materials Science Forum. Trans. Tech. Publications Ltd., 2000, 331: 197-202.

[150] TODA H, SINCLAIR I, BUFFIERE J Y, et al. Assessment of the fatigue crack closure phenomenon in damage-tolerant aluminium alloy by in-situ high-resolution synchrotron X-ray microtomography[J]. Philosophical Magazine, 2003, 83(21): 2429-2448.

[151] ROBERTSON S W, MEHTA A, PELTON A R, et al. Evolution of crack-tip transformation zones in superelastic Nitinol subjected to in situ fatigue: A fracture mechanics and synchrotron X-ray microdiffraction analysis[J]. Acta Materialia, 2007, 55(18): 6198-6207.

[152] WU S C, YU C, ZHANG W H, et al. Porosity induced fatigue damage of laser welded 7075-T6 joints investigated via synchrotron X-ray microtomography[J]. Science and Technology of Welding and Joining, 2015, 20(1): 11-19.

[153] DINGLEY D. A simple straining stage for the scanning electron microscope[J]. Micron, 1969, 1(2): 206-210.

[154] VEHOFF H, NEUMANN P. In situ SEM experiments concerning the mechanism of ductile crack growth $[\mathrm{J}]$. Acta Metallurgica, 1979, 27(5): 915-920.

[155] PONCHARAL P, WANG Z, UGARTE D, et al. Electrostatic deflections and electromechanical resonances of carbon nanotubes[J]. Science, 1999, 283(5407): 1513-1516. 
[156] MORANO R, STOCK S R, DAVIS G R, et al. X-ray microtomography of fatigue crack closure as a function of applied load in Al-Li 2090 T8E41 samples[J]. MRS Proceedings, 2011, 591: 31.

[157] FITZPATRICK M E, LODINI A. Analysis of residual stress by diffraction using neutron and synchrotron radiation[M]. London: Taylor \& Francis, 2003.

[158] LEE S Y, CHOO H, LIAW P K, et al. In situ neutron diffraction study of internal strain evolution around a crack tip under variable-amplitude fatigue-loading conditions[J]. Scripta Materialia, 2009, 60(10): 866-869.

[159] LOPEZ-CRESPO P, PERALTA J V, KELLEHER J F, et al. In situ through-thickness analysis of crack tip fields with synchrotron $\mathrm{X}$-ray diffraction[J]. International Journal of Fatigue, 2019， 127: 500-508.

[160] VANLANDUIT S, VANHERZEELE J, LONGO R, et al. A digital image correlation method for fatigue test experiments[J]. Optics and Lasers in Engineering, 2009, 47(3-4): 371-378.

[161] ZYBELL L, CHAVES H, KUNA M, et al. Optical in situ investigations of overload effects during fatigue crack growth in nodular cast iron[J]. Engineering Fracture Mechanics, 2012, 95: 45-56.

[162] LU Y W, LUPTON C, ZHU M L, et al. In situ experimental study of near-tip strain evolution of fatigue cracks[J]. Experimental Mechanics, 2015, 55(6) : 1175-1185.

[163] ZHU M L, LU Y W, LUPTON C, et al. In situ near-tip normal strain evolution of a growing fatigue $\operatorname{crack}[\mathrm{J}]$. Fatigue \& Fracture of Engineering Materials \& Structures, 2016, 39(8): 950-955.

[164] YANG B, VASCO-OLMO J M, DÍAZ F A, et al. A more effective rationalisation of fatigue crack growth rate data for various specimen geometries and stress ratios using the CJP model[J]. International Journal of Fatigue, 2018, 114: 189-197.

[165] 杨冰, JAMES M N. 基于 CJP 模型的疲劳裂纹扩展率 曲线及应用方法 $[\mathrm{J}]$. 机械工程学报, 2018, 54(18): 76-84.

YANG Bing, JAMES M N. Fatigue crack growth rate curve based on the CJP model and its application method[J]. Journal of Mechanical Engineering, 2018, 54(18): $76-84$.

[166] YIN Y, XIE H, HE W. In situ SEM-DIC technique and its application to characterize the high-temperature fatigue crack closure effect[J]. Science China Technological Sciences, 2020, 63(2): 265-276.

[167] CHEN R, ZHU M L, XUAN F Z, et al. Near-tip strain evolution and crack closure of growing fatigue crack under a single tensile overload[J]. International Journal of Fatigue, 2020， 134: 105478.

[168] HÄRKEGÅRD G. A finite element analysis of elastic-plastic plates containing cavities and inclusions with reference to fatigue crack initiation[J]. International Journal of Fracture, 1973，9(4): 437-447.

[169] 林晓斌. 基于有限元的疲劳设计分析系统 MSC/FATIGUE[J]. 中国机械工程, 1998(11): 12-16. LIN Xiaobin. MSC/FATIGUE design and analysis system based on finite element[J]. China Mechanical Engineering, 1998(11): 12-16.

[170] MCCLUNG R, SEHITOGLU H. On the finite element analysis of fatigue crack closure-1. Basic modeling issues[J]. Engineering Fracture Mechanics, 1989, 33(2): $237-252$

[171] HUANG Y. A user-material subroutine incroporating single crystal plasticity in the ABAQUS finite element program[M]. Cambridge, MA: Harvard University, 1991.

[172] ROTERS F, EISENLOHR P, HANTCHERLI L, et al. Overview of constitutive laws, kinematics , homogenization and multiscale methods in crystal plasticity finite-element modeling: Theory, experiments, applications[J]. Acta Materialia, 2010, 58(4): 1152-1211.

[173] ROTERS F, EISENLOHR P, BIELER T R, et al. Crystal plasticity finite element methods: in materials science and engineering[M]. Weinheim: John Wiley \& Sons, 2011.

[174] MCDOWELL D L. A perspective on trends in multiscale plasticity[J]. International Journal of Plasticity, 2010, 26(9): 1280-1309.

[175] SANGID M D, MAIER H J, SEHITOGLU H. A physically based fatigue model for prediction of crack initiation from persistent slip bands in polycrystals[J]. Acta Materialia, 2011, 59(1): 328-341.

[176] DUNNE F P E. Fatigue crack nucleation: Mechanistic modelling across the length scales[J]. Current Opinion in Solid State and Materials Science, 2014, 18(4): 170-179.

[177] LI D F, BARRETT R A, O'DONOGHUE P E, et al. A multi-scale crystal plasticity model for cyclic plasticity and low-cycle fatigue in a precipitate-strengthened steel at elevated temperature[J]. Journal of the Mechanics and 
Physics of Solids, 2017, 101: 44-62.

[178] SUKUMAR N, CHOPP D L, MORAN B. Extended finite element method and fast marching method for three-dimensional fatigue crack propagation[J]. Engineering Fracture Mechanics, 2003， 70(1): 29-48.

[179] KACHER J, ZHU T, PIERRON O, et al. Integrating in situ TEM experiments and atomistic simulations for defect mechanics[J]. Current Opinion in Solid State and Materials Science, 2019, 23(3): 117-128.

[180] LAVENSTEIN S, EL-AWADY J A. Micro-scale fatigue mechanisms in metals: Insights gained from small-scale experiments and discrete dislocation dynamics simulations[J]. Current Opinion in Solid State and Materials Science, 2019, 23(5): 100765.

[181] PYTTEL B, SCHWERDT D, BERGER C. Very high cycle fatigue-Is there a fatigue limit? [J]. International Journal of Fatigue, 2011， 33(1): 49-58.

[182] MUGHRABI H. On 'multi-stage'fatigue life diagrams and the relevant life-controlling mechanisms in ultrahigh-cycle fatigue[J]. Fatigue \& Fracture of Engineering Materials \& Structures, 2002, 25(8-9): 755-764.

[183] 朱明亮, 轩福贞. 材料疲劳极限的科学本质//10000 个 科学难题 (制造科学卷) [M]. 北京: 科学出版社, 2018 . ZHU Mingliang, XUAN Fuzhen. The nature of fatigue limit of materials// 10000 scientific problems (Manufacturing Science Volume)[M]. Beijing: Science Press, 2018

[184] MUGHRABI H, WANG R, DIFFERT K, et al. Fatigue crack initiation by cyclic slip irreversibilities in high-cycle fatigue[C]// Fatigue Mechanisms: Advances in Quantitative Measurement of Physical Damage. New York: ASTM International, 1983.

[185] SANGID M D. The physics of fatigue crack initiation[J]. International Journal of Fatigue， 2013， 57: 58-72.

[186] POLÁK J, MAN J. Experimental evidence and physical models of fatigue crack initiation[J]. International Journal of Fatigue, 2016，91: 294-303.

[187] MUGHRABI H. Microstructural mechanisms of cyclic deformation, fatigue crack initiation and early crack growth $[\mathrm{J}]$. Philosophical Transactions of the Royal Society A: Mathematical, Physical and Engineering Sciences, 2015, 373(2038): 20140132.

[188] 谢里阳, 徐影, 王德俊. 疲劳过程中材料强化和弱化 现象的探讨 $[\mathrm{J}]$. 机械强度，1991，13(1): 32-35.
XIE Liyang, XU Hao, WANG Dejun. Material strengthening and weakening phenomena in fatigue $[\mathrm{J}]$. Journal of Mechanical Strength, 1991, 13(1): 32-35.

[189] ZHANG Z, WANG Z. Grain boundary effects on cyclic deformation and fatigue damage $[\mathrm{J}]$. Progress in Materials Science, 2008, 53(7): 1025-1099.

[190] 范永升, 黄渭清, 杨晓光, 等. 某型航空发动机浴轮 叶片服役微观损伤研究 [J]. 机械工程学报, 2019, 55(13): 122-128.

FAN Yongsheng, HUANG Weiqing, YANG Xiaoguang, et al. Microstructural damage analysis of service turbine blades for an aero-engine[J]. Journal of Mechanical Engineering, 2019, 55(13): 122-128.

[191] ZHANG T, JIANG J, SHOLLOCK B A, et al. Slip localization and fatigue crack nucleation near a non-metallic inclusion in polycrystalline nickel-based superalloy $[\mathrm{J}]$. Materials Science and Engineering: A, 2015, 641: 328-339.

[192] WANG D Q, ZHU M L, XUAN F Z. Correlation of local strain with microstructures around fusion zone of a Cr-Ni-Mo-V steel welded joint[J]. Materials Science and Engineering: A, 2017, 685: 205-212.

[193] MILLER M P, PARK J S, DAWSON P R, et al. Measuring and modeling distributions of stress state in deforming polycrystals[J]. Acta Materialia, 2008, 56(15): 3927-3939.

[194] HERBIG M, KING A, REISCHIG P, et al. 3-D growth of a short fatigue crack within a polycrystalline microstructure studied using combined diffraction and phase-contrast X-ray tomography[J]. Acta Materialia, 2011, 59(2): 590-601.

[195] NARAGANI D P, SHADE P A, KENESEI P, et al. $\mathrm{X}$-ray characterization of the micromechanical response ahead of a propagating small fatigue crack in a Ni-based superalloy[J]. Acta Materialia, 2019, 179: 342-359.

[196] LIU S D, ZHU M L, ZHOU H B, et al. Strain visualization of growing short fatigue cracks in the heat-affected zone of a Ni-Cr-Mo-V steel welded joint: Intergranular cracking and crack closure[J]. International Journal of Pressure Vessels and Piping, 2019, 178: 103992.

[197] MORSDORF L, JEANNIN O, BARBIER D, et al. Multiple mechanisms of lath martensite plasticity[J]. Acta Materialia, 2016, 121: 202-214.

[198] RITCHIE R O. Mechanisms of fatigue-crack propagation 
in ductile and brittle solids[J]. International Journal of Fracture, 1999, 100(1): 55-83.

[199] ZHU M L, LU Y W, LUPTON C, et al. In situ near - tip normal strain evolution of a growing fatigue $\operatorname{crack}[\mathrm{J}]$. Fatigue \& Fracture of Engineering Materials \& Structures, 2016, 39(8): 950-955.

[200] WITHERS P. Fracture mechanics by three-dimensional crack-tip synchrotron X-ray microscopy[J]. Philosophical Transactions of the Royal Society A: Mathematical, Physical and Engineering Sciences, 2015，373(2036): 20130157.

[201] KING A, JOHNSON G, ENGELBERG D, et al. Observations of intergranular stress corrosion cracking in a grain-mapped polycrystal[J]. Science, 2008 , 321(5887): 382-385.

[202] 徐春广, 李培禄. 无应力制造技术 $[\mathrm{J}]$. 机械工程学报, 2020, 56(8): 113-132.

XU Chunguang, LI Peilu. Stress-free manufacturing technology[J]. Journal of Mechanical Engineering, 2020, 56(8): 113-132.

[203] 轩福贞, 宫建国. 基于损伤模式的压力容器设计原理 [M]. 北京: 科学出版社, 2020 .

XUAN Fuzhen, GONG Jianguo. Fundamental and approaches for damage mode-based design of pressure vessels[M]. Beijing: Science Press, 2020.

[204] ZHANG W C, ZHU M L, WANG K, et al. Failure mechanisms and design of dissimilar welds of $9 \% \mathrm{Cr}$ and
$\mathrm{CrMoV}$ steels up to very high cycle fatigue regime[J]. International Journal of Fatigue, 2018, 113: 367-376.

[205] ZHU M L, XUAN F Z. Failure mechanisms and fatigue strength reduction factor of a $\mathrm{Cr}-\mathrm{Ni}-\mathrm{Mo}-\mathrm{V}$ steel welded joint up to ultra-long life regime[C]/MATEC Web of Conferences, 12th International Fatigue Congress, FATIGUE 2018. 2018, 165: 21012.

[206] YANG Guangxue, WANG Meng, LI Qiang, et al. Methodology to evaluate fatigue damage of high-speed train welded bogie frames based on on-track dynamic stress test data[J]. Chinese Journal of Mechanical Engineering, 2019, 32(3): 193-200.

[207] KUSIAK A. Smart manufacturing must embrace big data[J]. Nature, 2017, 544(7648): 23-25.

[208] ZHU M L, XUAN F Z. Effect of microstructure on strain hardening and strength distributions along a Cr-Ni-Mo-V steel welded joint[J]. Materials \& Design, 2015, 65: 707-715.

作者简介: 轩福贞, 男, 1970 年出生, 博士, 教授。主要研究方向为机 械结构完整性。

E-mail: fzxuan@ecust.edu.cn

朱明亮, 男, 1984 年出生, 博士, 教授。主要研究方向为机械结构疲劳 与断裂。

E-mail: mlzhu@ecust.edu.cn

王国彪, 男, 1964 年出生, 博士, 教授。主要研究方向为现代设计理论 与方法、车辆工程等。

E-mail: gbwang@nsfc.gov.cn 